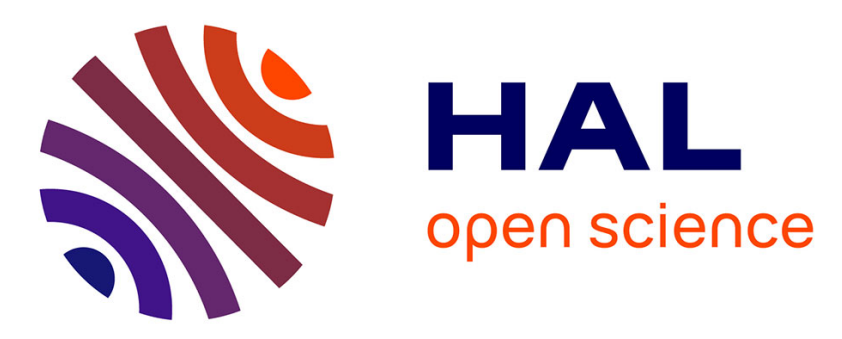

\title{
Tracking the variation of complex mode shapes for damage quantification and localization in structural systems
}

Maria Giovanna G Masciotta, Daniele Pellegrini

\section{- To cite this version:}

Maria Giovanna G Masciotta, Daniele Pellegrini. Tracking the variation of complex mode shapes for damage quantification and localization in structural systems. Mechanical Systems and Signal Processing, In press, 169 (108731). hal-03444118

\author{
HAL Id: hal-03444118 \\ https://hal.science/hal-03444118
}

Submitted on 23 Nov 2021

HAL is a multi-disciplinary open access archive for the deposit and dissemination of scientific research documents, whether they are published or not. The documents may come from teaching and research institutions in France or abroad, or from public or private research centers.
L'archive ouverte pluridisciplinaire HAL, est destinée au dépôt et à la diffusion de documents scientifiques de niveau recherche, publiés ou non, émanant des établissements d'enseignement et de recherche français ou étrangers, des laboratoires publics ou privés. 


\title{
Tracking the variation of complex mode shapes for damage quantification and localization in structural systems
}

\author{
M.G. Masciotta ${ }^{1,2 *}$, D. Pellegrini ${ }^{2}$ \\ ${ }^{1}$ Department of Engineering and Geology, University “G. d'Annunzio” of Chieti-Pescara, \\ Pescara, Italy. \\ ${ }^{2}$ Institute of Information Science and Technologies “'A. Faedo”, ISTI-CNR, Pisa, Italy \\ ${ }^{*}$ Corresponding Author: \\ Email: g.masciotta@unich.it \\ ORCID ID: https://orcid.org/0000-0003-2364-3490
}

\begin{abstract}
Real structures' mode shapes estimated by modal analysis techniques have a common feature: in most cases they are complex, and this complexity can derive from nonproportional damping, nonlinearities, mass loading effects, high modal density and localized damage, among others. Starting from the contributions available in the literature, the present paper investigates, from a numerical and experimental point of view, the correlation existing between localized damage and variation of global modal complexity indices conventionally employed to quantify the nonproportionality of damping in structural systems. Finally, driven by the inferences made through numerical and experimental test cases by tracking the variation of complex modes over multiple and progressive damage scenarios, a new index for damage localization and quantification is formulated and validated against real data.
\end{abstract}

Keywords: complex modal analysis, complex mode shapes, damage quantification, damage localization, structural health monitoring

\section{Introduction}

Structural systems can exhibit different vibration mode shapes, depending on the nature and distribution of energy dissipation mechanisms. The most common approach to characterize the system's damping responsible for the dissipative forces is to resort to a classical viscous damping model, known as Rayleigh damping. This model relies on the assumption that the damping matrix is a linear combination of the mass and stiffness matrices, thereby allowing to 
extend the classical modal analysis developed for undamped systems to damped structures. Yet, real-world systems consist of many substructures made up of different materials and tied up together in various fashions, often forming elaborated spatial geometries for which such assumption is sometimes constrictive and quite unrealistic [1]. In case of nonproportional damping, the equations of motion defining the direct problem in modal coordinates result to be coupled, thus mode shapes turn out to be described by complex quantities [2], [3].

Although modern dynamic identification methods are able to readily identify complex mode shapes, the use of real mode shapes continues to be the basis for the majority of modal analysis applications, including model calibration and updating, structural health monitoring and vibration-based damage identification [4]. Various techniques have been developed to extract real modes from complex eigenvectors, which is crucial to achieve an adequate correlation between experimental and analytical data [5], [6], [7], [8], [9], [10], [11], [12], [13]. But, ignoring the complexity of vibration modes estimated for real physical systems can sometimes lead to incorrect or misleading results in a field that is seeking for higher and higher accuracy [2], i.e. the field of structural identification, health monitoring and damage assessment. Structures experiencing local damages tend to exhibit out-of-phase vibrations because of the unsynchronized movements of the different nodes that do not pass through their maxima and minima at the same instant in time [13]-[14]. This mechanical phenomenon allows to understand why the imaginary part of the mode shapes can often provide more information about the spatial location and extent of damage as compared to its real counterpart.

In general, the inherent dynamic properties of a system are all strictly related to the physical and mechanical characteristics of the structure, but mode shapes are far more effective in localizing the damage than frequencies or damping alone [15]. Natural frequencies are easy to identify but they refer to the global structural behaviour and can result into the same amount of change even for different damage locations [16]; moreover, their sensitivity to mass variations and environmental conditions often introduces uncertainties in their use as unbiased damage indicators [17]. Conversely, although proven advantageous in some cases, damping values are highly affected by measurement errors and their estimates can show large scatters, being therefore rarely employed for damage assessment purposes [18], [19], [20]. To implicitly account for information associated with nonproportional damping arising from local damage phenomena, the scientific community has recently shifted the focus of its research activity towards the effects that non-uniform energy dissipation mechanisms cause to the mode shapes of a vibrating system. The hypothesis underlying this research trend is that undamaged structures exhibit proportional damping and their eigenmodes can be reduced to the real ones 
of the corresponding ideal undamped systems, whereas damaged structures exhibit nonproportional damping and their eigenmodes are lists of complex quantities associated to the amount of damage present in the system [21].

Based on these considerations, over the last decades several contributions have spread in the literature trying to verify the relationship between damage and modal complexity in order to exploit this information for structural health monitoring of existing artefacts. Worth of mention are the pioneering work of Kawiecki [18], who tried to use the modal damping characteristics of a tested structure for damage detection; the investigations carried out by Iezzi et al. [22], [23], [24], who applied and validated the effectiveness of a measurable complexity index to detect the presence of damage in framed structures having a typical "shear-type" behaviour; the study of Masciotta et al. [14], who embarked on the analysis of the real and imaginary components of complex eigenvectors for damage localization; and the very recent study of Lofrano et al. [21], who proposed a numerical approach based on a perturbation method to detect, locate and quantify the damage in framed structures by exploiting state-of-the-art complexity indices. Despite the scientific robustness of the developed approaches and the consensus reached on the pivotal role that complex eigenmodes can play as a measure of structural damage, the applicative counterparts have shown some limitations. Most of these works assume that the increase in mode shapes complexity comes only from the energy dissipated along with damage occurrence. However, as Deblauwe and Allemang highlighted in [25], the imaginary content of modal vectors experimentally identified can also increase due to aliasing, leakage, mass loading effects, measurements noise, nonlinearities, high modal density, and identification errors. It follows that, although a correlation may exist between presence of damage and amount of complexity in damped systems, this is not univocal, especially if the origin of such a complexity is simultaneously linked to factors other than damage. Notwithstanding the significant number of papers published on complex modes since the original contribution of Caughley and O'Kelley [26] and the degree of maturity reached by modern dynamic identification techniques, the scientific community is still reluctant in transferring complex mode theory to practical applications, indicating that this subject is not yet fully understood.

The present work is rooted in the research framework outlined above and aims to analyse the complex nature of mode shapes in damaged systems featuring nonproportional damping from a numerical and experimental point of view. To this end, different sets of modal data are generated through numerical simulations, allowing a first discussion on the use of state-of-theart modal complexity indices for damage identification purposes. Then, a dense set of 
experimental data from ambient vibration tests is employed to show the limitations of existing theories and indicators, and to introduce a new index for damage quantification and localization based on the weighted componentwise difference of the imaginary content of complex modes. The next sections of the papers are organized as follows. Section 2 recalls concepts related to the modal analysis of damped multi-degree-of-freedom systems as well as the measurable indices employed to address the problem of damage detection using complex modes. Section 3 analyses the complexity of eigenmodes from a numerical point of view by resorting to two different examples of damped structures: a beam subjected to progressive damage scenarios in a single location and a segmental arch subjected to progressive damage scenarios in multiple locations. Section 4 provides a thorough examination of the problem of modal complexity from an experimental point of view, which ultimately leads to a pointwise damage analysis of the relative variation of complex-valued modal vectors and to the proposal of a damage indicator that combines natural frequencies and complex eigenmodes, to detect, locate and assess the damage and its evolution path over progressive and multiple scenarios. Finally, Section 5 summarizes the main conclusions of the work.

\section{Modal analysis of damped structures}

Real structures are continuous and nonhomogeneous systems having an infinite number of degrees of freedom (DOFs). Usually, they are analysed resorting to a discretization that makes use of a finite number of DOFs, as many as necessary to guarantee plenty of precision. In general, the second-order differential equations of motion describing the dynamic behaviour of a multi-degree-of-freedom system (MDOF), if written in matrix form, can be efficiently solved using the mode shapes and turning them into modal coordinates. Because of mode shape orthogonality with respect to the mass and stiffness matrices, the modal coordinate mass and stiffness matrices will be diagonal. In the case of proportional damping, the transformation will also produce a diagonal damping matrix in modal coordinates, giving rise to classical normal modes (i.e. real mode shapes). Otherwise, the equations will be coupled, and the problem must be addressed in a different way. The following subsection recalls the main concepts of the nonclassical problem of complex modes and the method used to solve it, which will be subsequently employed in Section 3 for the analysis of modal complexity through numerical data. 


\subsection{State-space formulation}

Let us consider a linear elastic underdamped MDOF structure discretized into finite elements, the second-order differential equations governing the free vibration problem are expressed by:

$$
\mathrm{Mü}(\mathrm{t})+\mathrm{Cu}(\mathrm{t})+\mathrm{Ku}(\mathrm{t})=0
$$

where $\mathrm{u}$ is the displacement vector, which belongs to $\mathrm{R}^{n}$ and depends on time $\mathrm{t}$; $\mathrm{u}$ is the second derivative of $\mathrm{u}$ with respect to time $\mathrm{t} ; \mathrm{M}, \mathrm{C}$, and $\mathrm{K} \in \mathrm{R}^{n \times n}$ are the mass, viscous damping and stiffness matrices of the finite-element assemblage. Displacements $u_{i}$ are also called degrees of freedom and the integer $n$ corresponds to the total number of degrees of freedom of the structure. $\mathrm{K}$ is symmetric and positive-semidefinite, $\mathrm{M}$ is symmetric and positive-definite, and both are banded with bandwidth depending on the numbering of the finite-element nodal points.

Regarding the viscous damping matrix C, if the following conditions are met [26]:

$$
\mathrm{CM}^{-1} \mathrm{~K}=\mathrm{KM}^{-1} \mathrm{C}=\left(\mathrm{CM}^{-1} \mathrm{~K}\right)^{\mathrm{T}}
$$

the system has classical normal modes (real mode shapes); i.e., the mode shapes of the undamped system uncouple the damped equations of motion [27] and the Eq. (1.1) is turned into a real constrained generalized eigenvalue problem.

If the conditions in Eq. (1.2) are not satisfied, the not proportional viscous damping must be considered in the computation of the system modes, which then produces complex mode shapes. By assuming that:

$$
\mathrm{u}(\mathrm{t})=\phi \mathrm{e}^{\mathrm{st}}
$$

with $\phi$ a vector of $\mathrm{R}^{n}$ and $\mathrm{s}$ a complex number, and applying the modal superposition [28], the Eq. (1.1) is transformed into a complex polynomial eigenvalue problem [29]:

$$
\left[\mathrm{s}^{2} \mathrm{M}+\mathrm{sC}+\mathrm{K}\right] \phi=0
$$

The simplest way to solve this kind of problem, considering a generic non-proportional viscous damping, is to make use of the state space formulation casting the equations of motion in the first-order form. Defined the complex vector w(t) as:

$$
\mathrm{w}(\mathrm{t})=\left[\begin{array}{c}
\mathrm{u}(\mathrm{t}) \\
\dot{\mathrm{u}}(\mathrm{t})
\end{array}\right]
$$

Equation (1.1) turns into Equation (1.6):

\footnotetext{
${ }^{1}$ where $(\cdot)^{-1}$ and $(\cdot)^{\mathrm{T}}$ are respectively the inverse and transposed matrices.
} 


$$
A \dot{w}(t)+B w(t)=0
$$

where $\mathrm{A}$ and $\mathrm{B}$ are $2 n \times 2 n$ symmetric matrices defined as:

$$
A=\left[\begin{array}{cc}
C & M \\
M & 0
\end{array}\right], B=\left[\begin{array}{cc}
K & 0 \\
0 & -M
\end{array}\right]
$$

Substituting the assumed solution (1.3) and its time derivative into Eq. (1.5),

$$
\mathrm{w}(\mathrm{t})=\left[\begin{array}{c}
\phi \\
\mathrm{s} \phi
\end{array}\right] \mathrm{e}^{\mathrm{st}}=\mathrm{z} \mathrm{e}^{\mathrm{st}}, \dot{\mathrm{w}}(\mathrm{t})=\left[\begin{array}{c}
\mathrm{s} \phi \\
\mathrm{s}^{2} \phi
\end{array}\right] \mathrm{e}^{\mathrm{st}}=\mathrm{s} \mathrm{z} \mathrm{e}^{\mathrm{st}}
$$

and since se $\mathrm{s}^{\mathrm{st}}$ takes on nonzero values, it follows that:

$$
[\mathrm{sA}+\mathrm{B}] \mathrm{z}=0
$$

Equation (1.8) yields $2 n$ eigenvalues and associated eigenvectors; because, for a constrained system, A and B are real symmetric matrices, the eigenvalues result in complex conjugate pairs, with the corresponding eigenvectors also occurring in complex conjugate pairs [27]. The generic complex eigenvalue, distinguished by a negative real part for a stable system, has the following form:

$$
s_{j}=-\zeta_{j} \omega_{n, j} \pm i \omega_{n, j} \sqrt{1-\zeta_{j}^{2}}=-\zeta_{j} \omega_{n, j} \pm i \omega_{d, j}
$$

where $\omega_{\mathrm{n}, \mathrm{j}}$ and $\omega_{\mathrm{d}, \mathrm{j}}$ represent the $\mathrm{j}^{\mathrm{th}}$ natural and damped circular frequency of the system, $\mathrm{i}$ the imaginary unit and $\zeta_{\mathrm{j}}<1$ the $\mathrm{j}^{\text {th }}$ damping ratio; known the $\mathrm{j}^{\text {th }}$ eigenvalue, the corresponding natural circular frequency and damping ratio can be evaluated exploiting the expressions below:

$$
\begin{gathered}
\omega_{\mathrm{n}, \mathrm{j}}=\left|\mathrm{s}_{\mathrm{j}}\right| \\
\zeta_{\mathrm{j}}=\frac{-\operatorname{Re}\left(\mathrm{s}_{\mathrm{j}}\right)}{\omega_{\mathrm{n}, \mathrm{j}}}
\end{gathered}
$$

Introducing in Eq. (1.8) the eigenvalues recovered by the expression (1.9), it is possible to achieve $\mathrm{z}$ and then estimate the mode shapes of the system.

\subsection{Mode shape complexity for damage identification}

Non-uniform energy dissipation mechanisms due to non-proportional damping distribution within the structure lead to complex vibration modes. As a result, many indices based on the mode shapes complexity have been formulated in the literature to measure the damping nonproportionality of a vibrating system through experimental or operational modal analyses [3], [13], [24], [30]. Some of these indices have also been used to numerically estimate the damage evolution path in framed structures with a typical shear-type behaviour [21], assuming the 
mode complexity as a measure of structural damage. Among them, the most recurrent indicators are briefly recalled below along with their explicit expressions:

- Modal Imaginary Ratio (MIR). This index weighs the imaginary part of the complex mode shape with respect to its overall length:

$$
\operatorname{MIR}=\frac{\left\|\operatorname{Im}\left(\phi_{\mathrm{j}}\right)\right\|}{\left\|\phi_{\mathrm{j}}\right\| \|}
$$

- Modal dispersity (MD). This index provides the componentwise average of the imaginary part of the mode shape, namely its degree of scatter:

$$
\mathrm{MD}=\frac{\sum_{\mathrm{k}=1}^{n}\left|\operatorname{Im}\left(\phi_{\mathrm{jk}}\right)\right|}{n}
$$

- Modal Phase Difference (MPD). This index measures the maximum out of phase of the complex mode shape, viz. its angular dispersion:

$$
\operatorname{MPD}=\frac{\left|\theta_{\mathrm{j}, \max }\right|-\left|\theta_{\mathrm{j}, \min }\right|}{\pi}
$$

- Modal Polygon Area (MPA). This index quantifies the modal polygon area defined by the mode shape over the maximum modal polygon area:

$$
\mathrm{MPA}=\frac{A_{\mathrm{j}}}{\mathrm{n} A_{\mathrm{j}, \max }}, A_{\mathrm{j}, \max }=\mathrm{n} \cos \left(\frac{\pi}{\mathrm{n}}\right) \sin \left(\frac{\pi}{\mathrm{n}}\right)
$$

Note that in the previous Eqs. $\|\cdot\|$ indicates the Euclidean 2-norm operator and $|\cdot|$ means absolute value; $\operatorname{Im}(\cdot)$ is the imaginary part of the mode shape; $\phi_{\mathrm{j}}$ stands for the $\mathrm{j}^{\text {th }}$ natural mode shape; $\phi_{\mathrm{jk}}$ is the $\mathrm{k}^{\text {th }}$ component of $\phi_{\mathrm{j}}$; $n$ indicates the number of degrees of freedom of the structure; $\theta_{\mathrm{j}, \max }, \theta_{\mathrm{j}, \min }$ are the maximum and minimum phase angle of $\phi_{\mathrm{j}}$, respectively; $A_{\mathrm{j}}$ is the area enclosed by the polygon constituted by the components of $\phi_{j}$ in the complex plane, whereas $A_{j, m a x}$ is the maximum potential area of the modal polygons. 
The indices defined above are always evaluated after removing the dummy, or fictitious, complexity typical of natural modes obtained by state-space dynamic analysis. This is done through a normalization and rotation process [3] which consists in rotating the straight line representing the best linear fit of the mode shape in the complex plane, thus making the real part of the mode a maximum and the imaginary part a minimum. By definition, these indices are positive (except for MPD) and range between zero, when damping is proportional or null and the modes are exactly real-valued monophase vectors, and one, when damping is nonproportional, and the modes are fully complex vectors, meaning that all the points of the structure do not pass through their undeflected position at the same instant in time but feature a certain lag.

Two other coefficients enabling to relate pairs of mode shapes and quantify their level of complexity are also largely employed in the literature:

- Modal Phase Collinearity (MPC). This parameter, whose value ranges from zero to one (being equal to one for real vectors), is associated with the variance and covariance of the real $\operatorname{Re}(\cdot)$ and imaginary $\operatorname{Im}(\cdot)$ parts of an eigenvector, as specified below [31]:

$$
\begin{aligned}
& \text { MPC }=\left(\frac{\alpha_{1}-\alpha_{2}}{\alpha_{1}+\alpha_{2}}\right)^{2} \\
& \alpha_{1,2}=\frac{S_{x x}+S_{y y}}{2} \pm S_{x y} \sqrt{1+\left(\frac{S_{y y}-S_{x x}}{2 S_{x y}}\right)^{2}} \\
& S_{x x}=\operatorname{Re}\left(\phi_{j}\right)^{\mathrm{T}} \operatorname{Re}\left(\phi_{j}\right), S_{y y}=\operatorname{Im}\left(\phi_{j}\right)^{T} \operatorname{Im}\left(\phi_{j}\right), S_{x y}=\operatorname{Re}\left(\phi_{j}\right)^{\mathrm{T}} \operatorname{Im}\left(\phi_{j}\right)
\end{aligned}
$$

- Modal Assurance Criterion (MAC). This parameter quantifies the degree of correlation between two different mode shapes according to the following expression [32]:

$$
\operatorname{MAC}\left(\phi_{\mathrm{j}}, \phi_{\mathrm{m}}\right)=\frac{\left|\phi_{\mathrm{j}}^{*} \phi_{\mathrm{m}}\right|^{2}}{\left\|\phi_{\mathrm{j}}\right\|^{2}\left\|\phi_{\mathrm{m}}\right\|^{2}},\left\|\phi_{\mathrm{j}}\right\|,\left\|\phi_{\mathrm{m}}\right\| \neq 0
$$

where $(\cdot) *$ denotes the complex conjugate transpose of the mode shape. This coefficient, conventionally used to perform pairing between sets of modes, is a scalar varying between zero (no correlation) and one (full correlation) and is well-suited to the analysis of monophase vectors. 
In real situations, typical complex modes are somewhere in between the completely complex and the real modes [33]. Therefore, conventional modal analysis methods based on the assumption of normal (or nearly normal) mode shapes cannot be universally regarded as accurate and reliable for all applications. Modal complexity can originate from a variety of reasons, including damage, thus its correct quantification cannot be neglected.

\section{Analysis of modal complexity using numerical data}

In order to explore the complex nature of the mode shapes in systems with non-proportional damping arising from stiffness degradation, as well as to investigate the performance and effective damage-dependent variation of the indices reported in the previous section, two artificial examples were numerically generated (see Figure 1) and analysed: a pinned beam and a segmental arch on fixed supports. In either case, complex modal analyses were conducted on the undamaged and damaged configuration of the structure, focusing on the componentwise changes undergone by the imaginary part of the mode shapes with progressive damage scenarios. Indeed, very little attention has been paid in the literature to this particular aspect. The analyses carried out consisted of the following steps:

- Step 1 (reference scenario, RS): a preliminary modal analysis is performed to evaluate the natural frequencies and mode shapes of the undamaged system, assuming the damping matrix $\mathrm{C}$ as a linear combination of $\mathrm{K}$ and $\mathrm{M}$ [34];

- Step 2 (damage scenario, DS): localized damage is simulated in the structure by reducing the elastic modulus of some elements and the new stiffness matrix $\bar{K}$ of the damaged system is evaluated;

- Step 3: the complex eigenvalue problem in Eq. (1.4) is solved by the state space formulation (1.8), using the damaged stiffness matrix $\bar{K}$ in place of $K$, and assuming $C$ and $\mathrm{M}$ as constant and equal to the matrices computed in Step 1 for the initial undamaged state (basically the damping non-proportionality only arises as an indirect effect of the stiffness loss);

- Step 4: the frequency decay of the main vibration modes is estimated over progressive damage scenarios along with the MAC, MPC, MIR, MD, MPD and MPA indices;

- Step 5: the real and imaginary components of the mode shapes are plotted, and their changes analysed from the reference scenario up to the last damaged configuration. 
The simulations were performed combining the use of MATLAB R2018b and NOSA-ITACA code. The latter is a non-commercial FE code developed by ISTI-CNR [35]; thanks to the numerical method therein implemented to solve the FE model updating problem [36], [37], [38], it was possible to obtain the automatic extraction of the $\mathrm{M}, \mathrm{K}$ and $\overline{\mathrm{K}}$ matrices employed in step 3.
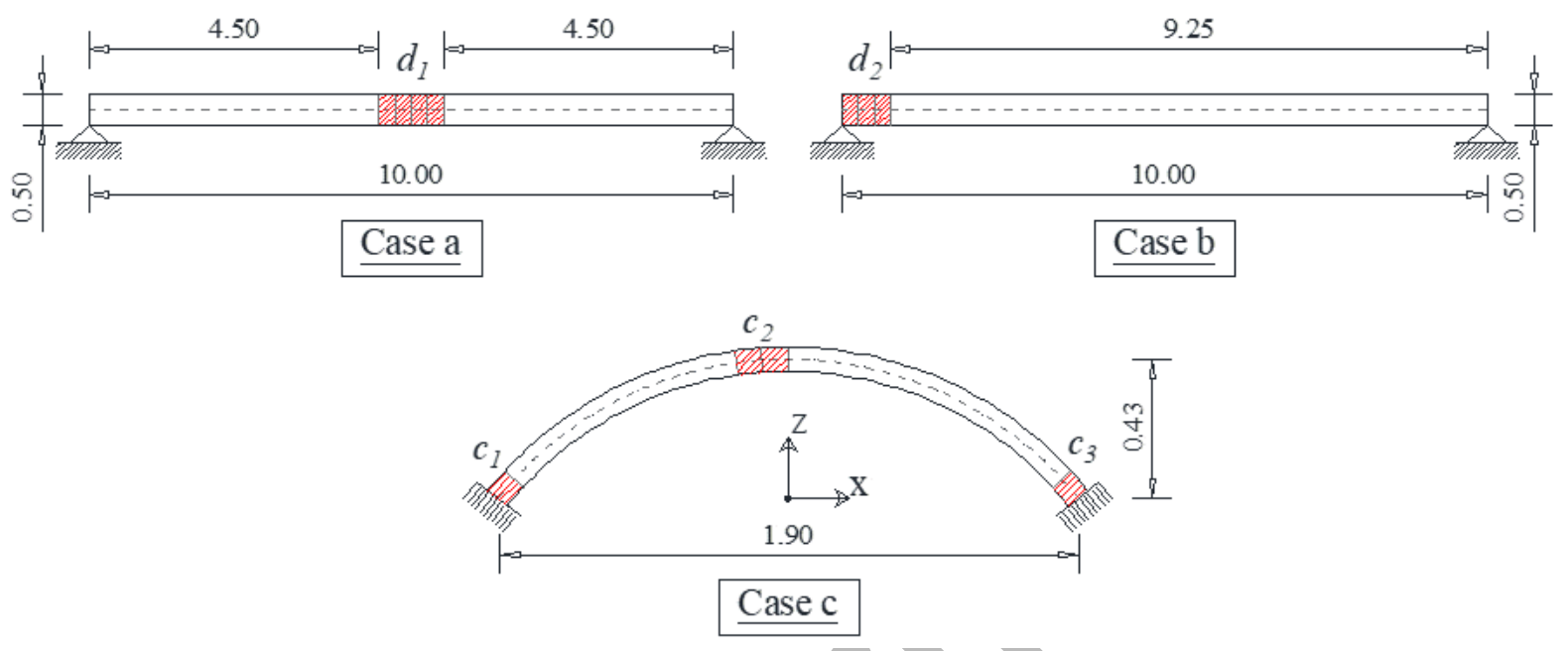

Figure 1. Geometry of the damped structures used in the numerical simulations (length in meters) with identification of the damage location(s).

\subsection{Damped beam under progressive damage scenarios with single damage location}

The first numerical example focused on the beam structure sketched in Figure 1. The beam featured a $10 \mathrm{~m}$ length and a square cross-section of $0.5 \mathrm{~m}$ side, it was pinned at the ends and forced to move in the $\mathrm{x}-\mathrm{z}$ plane. The system was discretized into 40 straight beam elements (element no. 9 of the NOSA-ITACA library [39]) for a total of 41 nodes and 246 degrees of freedom, allowing to obtain a simple yet refined baseline model far different from the reduced order models usually adopted in the literature to address the problem of modal complexity [23], [24].

In the first stage, a preliminary modal analysis was performed to evaluate the frequencies and mode shapes of the beam in the reference scenario (RS), under the assumption of homogeneous material with Young's modulus E=3.00 GPa, Poisson's ratio $v=0.2$ and mass density $\rho=1800$ $\mathrm{kg} / \mathrm{m}^{3}$. The first four natural frequencies obtained with the above parameters are reported in Figure 2 together with the corresponding mode shapes. Note that, dealing with a 2D problem, the main vibration modes of the structure are all vertical bending modes, and their sequential 
number reflects the number of curvatures (or the number of inflection points decreased by one unit) in the deflected beam shape.

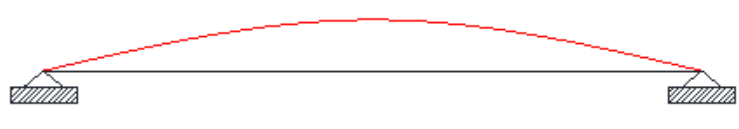

$1^{\text {st }}$ mode shape, $f_{1 \mathrm{i}}=2.92 \mathrm{~Hz}$

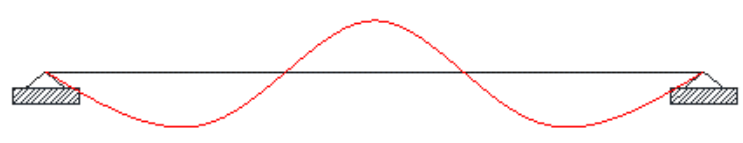

$3^{\text {rd }}$ mode shape, $f_{3 \mathrm{i}}=25.79 \mathrm{~Hz}$

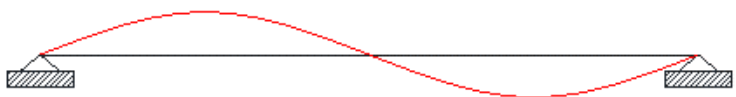

$2^{\text {nd }}$ mode shape, $f_{2 \mathrm{i}}=11.60 \mathrm{~Hz}$

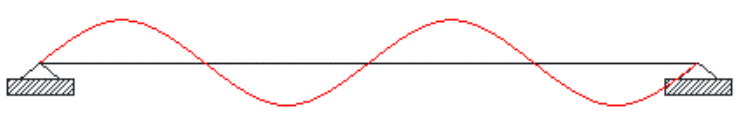

$4^{\text {th }}$ mode shape, $f_{4 \mathrm{i}}=45.17 \mathrm{~Hz}$

Figure 2. The first four vibration modes of the beam in the reference scenario.

Once extracted the stiffness and mass matrices from the NOSA-ITACA code, the damping matrix $\mathrm{C}$ was calculated under the well-known Rayleigh assumption given by:

$$
\begin{aligned}
& C=a_{0} M+a_{1} K \\
& a_{1}=\frac{2 c}{\omega_{n 1}+\omega_{n 2}}, \quad a_{0}=a_{1} \omega_{n 1} \omega_{n 2}
\end{aligned}
$$

where $\omega_{\mathrm{n} 1}$ and $\omega_{\mathrm{n} 2}$ are the first two natural circular frequencies of the undamped system and $\mathrm{c}$ is the damping ratio (taken as $3 \%$ for the present case).

Afterwards, damage (damage scenario, DS) is inserted in the beam middle part ( $d_{1}$, case $a$ ) and at the left end ( $d_{2}$, case b). Five damage scenarios (DS1-DS5) were progressively reached in each case by decreasing the elastic modulus of four (case $a$ ) and three (case $b$ ) elements from $10 \%$ to $50 \%$. For every DS the damaged stiffness matrix $\overline{\mathrm{K}}$ was evaluated by the NOSAITACA code and subsequently used to solve the complex eigenvalue problem.

The predicted natural frequencies and associated modal complexity levels obtained for case $a$ ) are summarized in Figure 3 and Figure 4 across the different DSs. As expected, frequencies result to be highly sensitive to structural damage: in fact, in the scenario characterized by a $50 \%$ reduction of the Young's modulus at mid-span (DS5), maximum frequency downshifts of 8.6\% and $6.3 \%$ are estimated for the first and third modes, respectively, which are the modes mainly affected in terms of global stiffness by the central location of the damage. On the contrary, even modes feature a null inflection point passing through the damage position, hence their low sensitivity to global stiffness changes and local deflection changes (the lower the mode, the lower its sensitivity to mode shape variations and vice versa). 
Concerning the complexity indices, it is worth noting that despite their different magnitude scales, they all exhibit similar trends (except for MPD), reading progressive variations consistent with the damage severity of each scenario but unable to catch the major influence on the first and third mode shape. Overall, the results highlight that: (i) MPC is minimally affected by the damage showing a maximum variation of barely $0.11 \%$ in the worst scenario; (ii) MPD index does provide no useful information on the damage evolution; (iii) MIR, MD and MPA seem very sensitive to damage (in particular for higher-order mode shapes) but monotonically increase with the damage evolution regardless of the different impact caused to the stiffness and deflection characteristics of each mode. Furthermore, none of the indicators gives spatial information about the damage position.

Figure 5 shows the pointwise evolution of the real and imaginary components of the four estimated mode shapes over the different damage scenarios. It is worth noting that, while the shifts in the real part are almost undetectable, or even non-existent in correspondence of the central damaged elements, the imaginary component is very sensitive to damage, as proved by the quantities $\Delta$ and $\Delta^{*}$ reported in the same figure and calculated as:

$$
\begin{aligned}
& \Delta=\left|\frac{\operatorname{Re}\left(\phi_{\mathrm{ij}}{ }^{\mathrm{DS} 5}\right)-\operatorname{Re}\left(\phi_{\mathrm{ij}}{ }^{\mathrm{RS}}\right)}{\operatorname{Re}\left(\phi_{\mathrm{ij}}{ }^{\mathrm{RS}}\right)}\right| \\
& \Delta^{*}=\left|\frac{\operatorname{Im}\left(\phi_{\mathrm{ij}}{ }^{\mathrm{DS} 5}\right)-\operatorname{Im}\left(\phi_{\mathrm{ij}}{ }^{\mathrm{DS} 1}\right)}{\operatorname{Im}\left(\phi_{\mathrm{ij}}{ }^{\mathrm{DS} 1}\right)}\right|
\end{aligned}
$$

In terms of average magnitude variation of the imaginary content, the higher the number of inflection points of the mode, the greater the componentwise shifts, resulting into a major number of false positives.
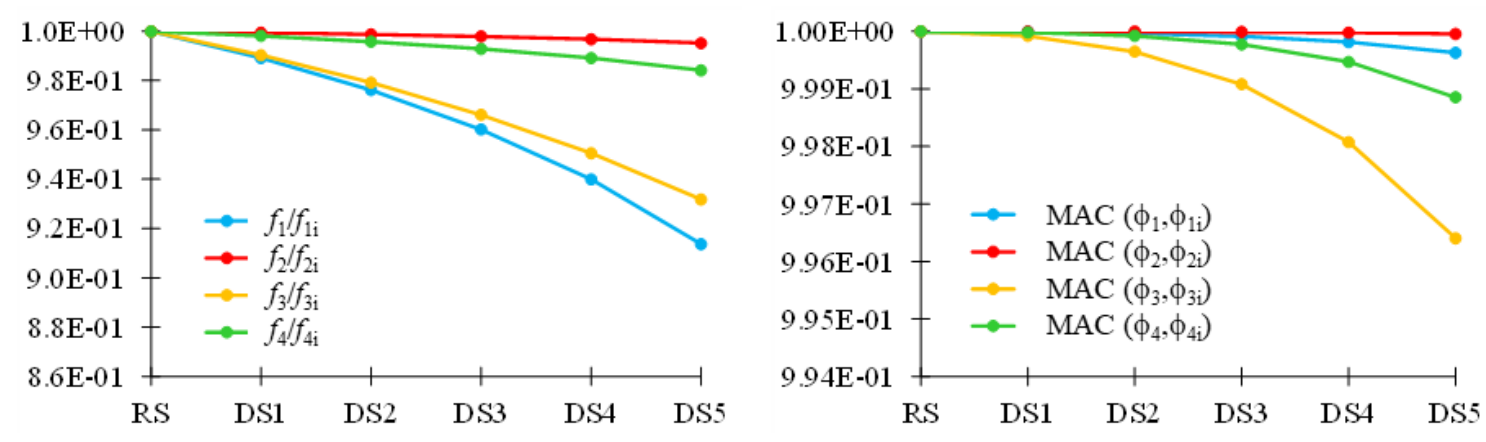

Figure 3. Case a): frequency decay and MAC trend versus damage scenario (DS). (For the interpretation of colours in the figure legend, the reader is referred to the web version of this article). 

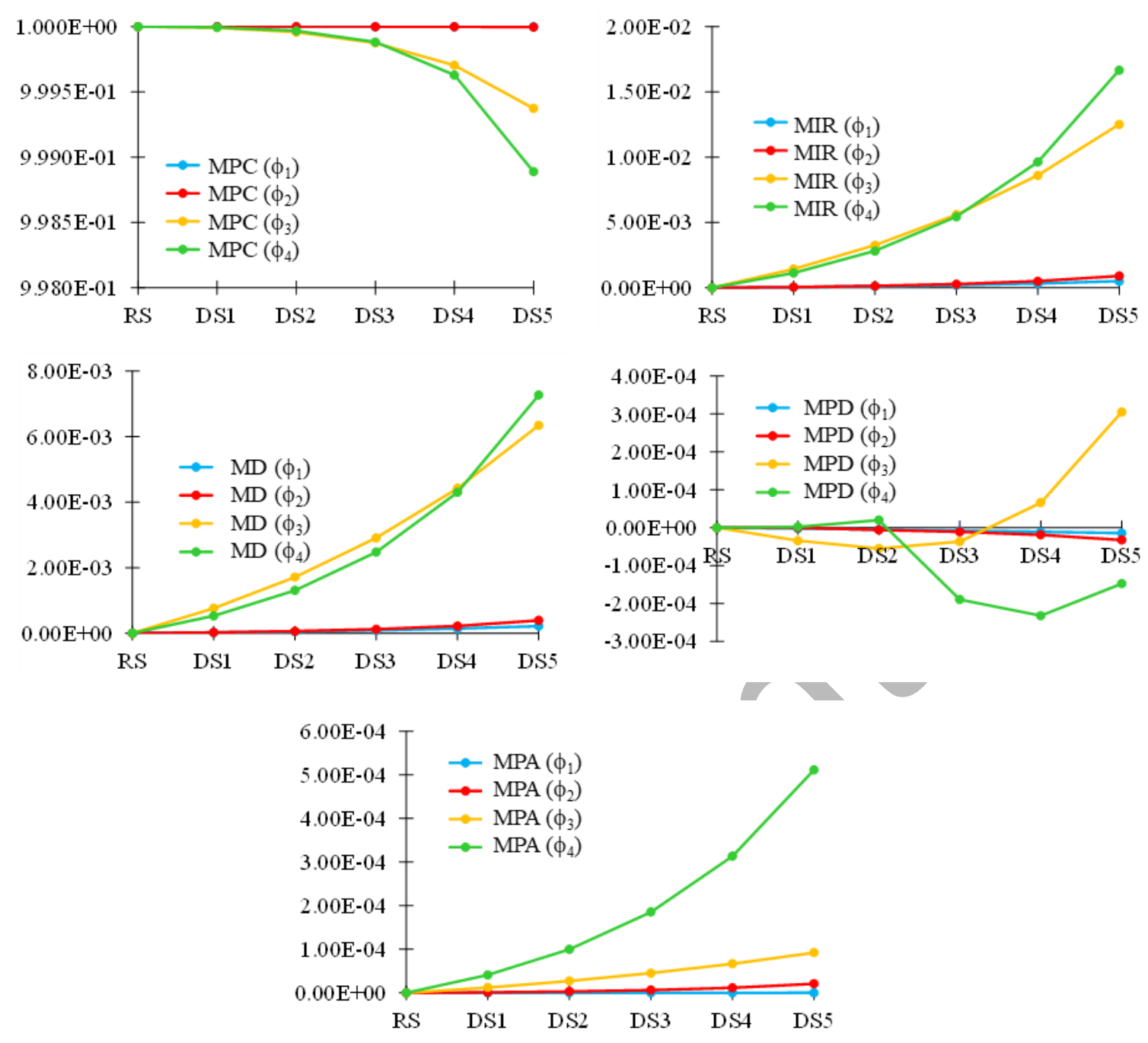

Figure 4. Case a): MPC, MIR, MD, MPD and MPA trend versus damage scenarios (DS). (For the interpretation of colours in the figure legend, the reader is referred to the web version of this article). 


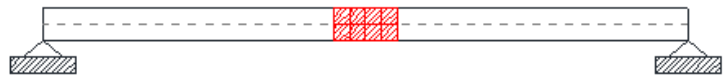

$1^{\text {st }}$ mode: real part

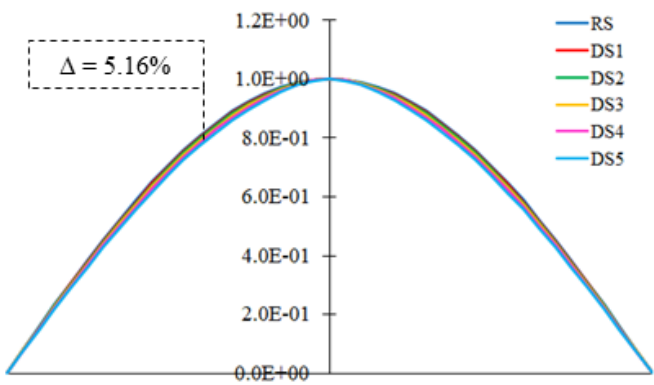

$2^{\text {nd }}$ mode: real part

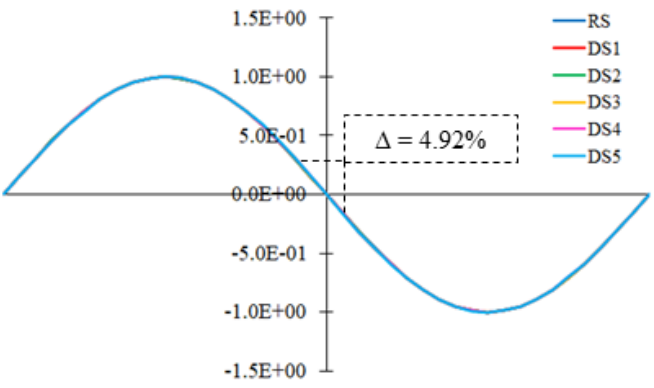

$3^{\text {rd }}$ mode: real part
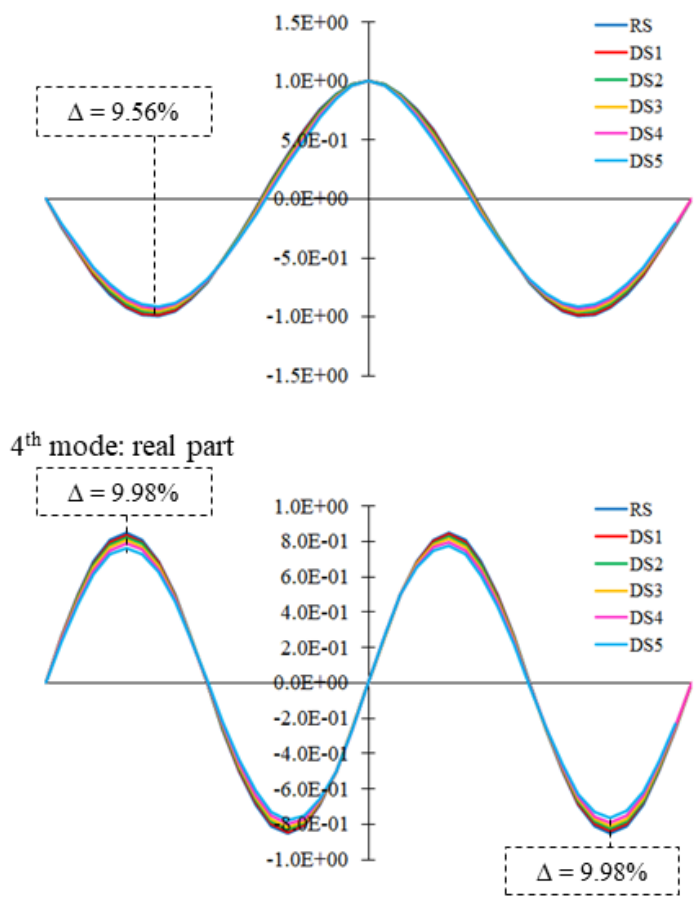

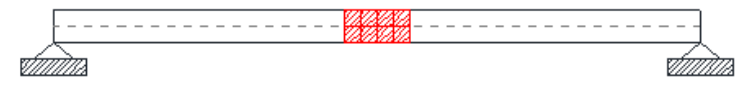

$1^{\text {st }}$ mode: imaginary part
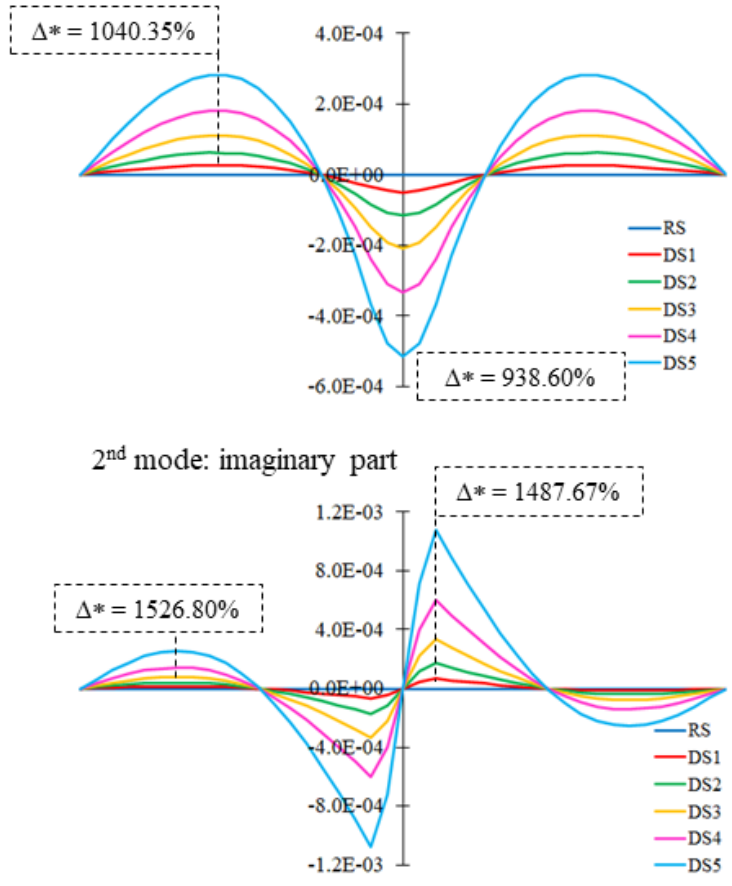

$3^{\text {rd }}$ mode: imaginary part

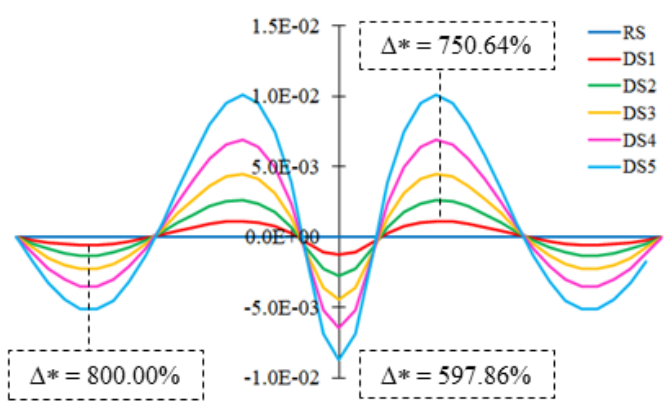

$4^{\text {th }}$ mode: imaginary part

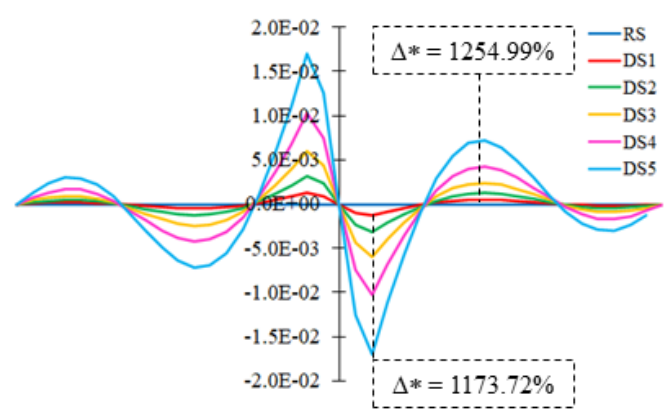

Figure 5. Case a): real component (on the left), and imaginary component (on the right) of the four mode shapes, throughout damage scenarios. (For the interpretation of colours in the figure legend, the reader is referred to the web version of this article). 
To better explore the correlation between damage location and mode complexity, the same analyses carried out for case $a$ ) are repeated for case $b$ ), namely for the beam structure where damage is localized at the left end, close to the pinned support. The predicted results, shown in Figure 6 and Figure 7, point out the greater impact that one-sided stiffness losses have on the higher-frequency modes of the beam and highlight the sensitivity of all complexity indices to mode complexification caused by unilateral damage scenarios, displaying increasing trends as the severity of the damage increases. Concerning the componentwise comparison of the real and imaginary parts illustrated in Figure 8, it is interesting to note how the magnitude of the mode shape imaginary component amplifies with progressive damage and reaches its maximum value near the damage position, unlike the real counterpart. This is particularly evident by comparing the imaginary component of the first mode shape of case $a$ ) with the corresponding one of case $b$ ). However, the average magnitude variation of the imaginary content of each mode shape does increase with the number of relevant inflection points, thus leading to false positives for higher-frequency modes.
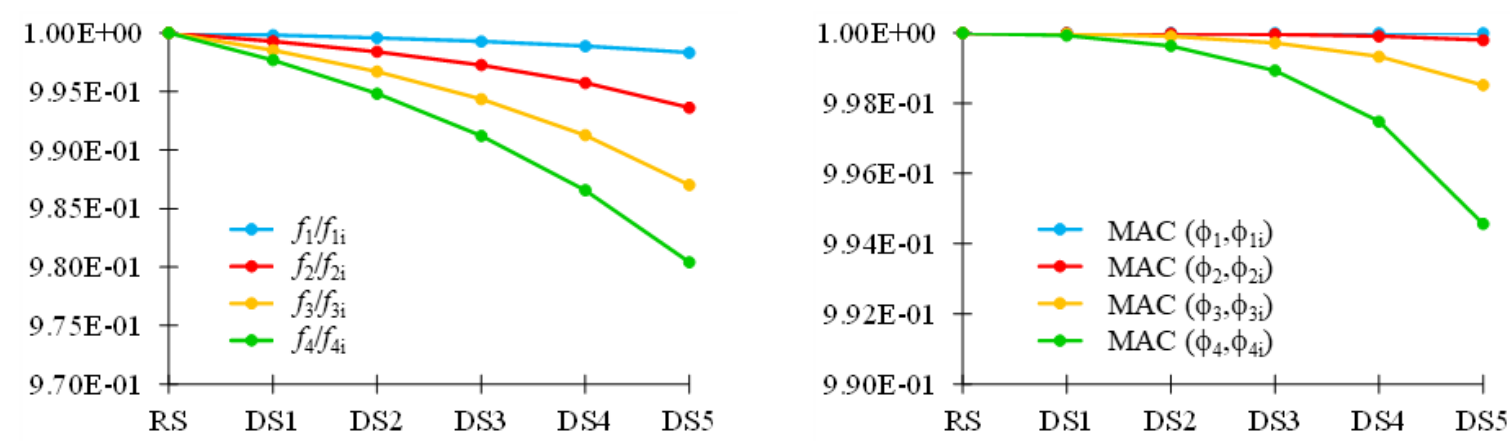

Figure 6. Case b): frequencies decay and MAC trend versus damage scenario (DS). (For the interpretation of colours in the figure legend, the reader is referred to the web version of this article). 

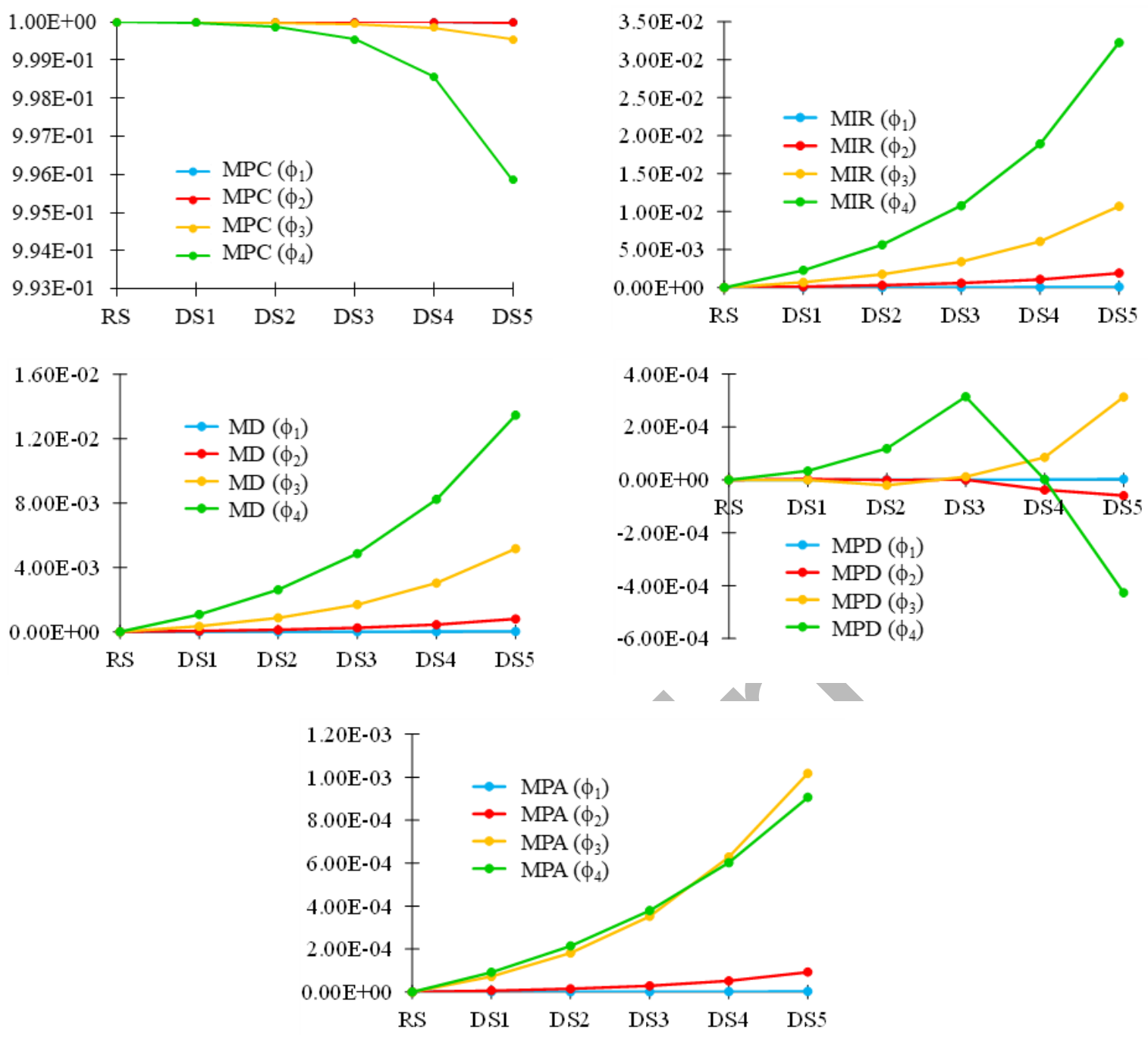

Figure 7. Case b): MPC, MIR, MD, MPD and MPA trend versus damage scenarios (DS). (For the interpretation of colours in the figure legend, the reader is referred to the web version of this article). 


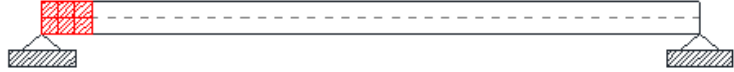

$1^{\text {st }}$ mode: real part

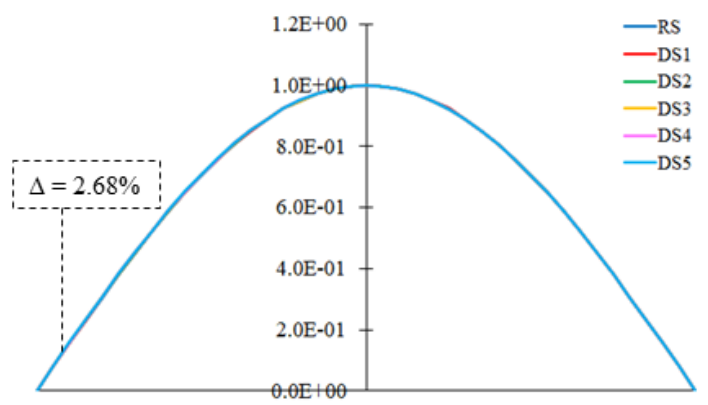

$2^{\text {nd }}$ mode: real part

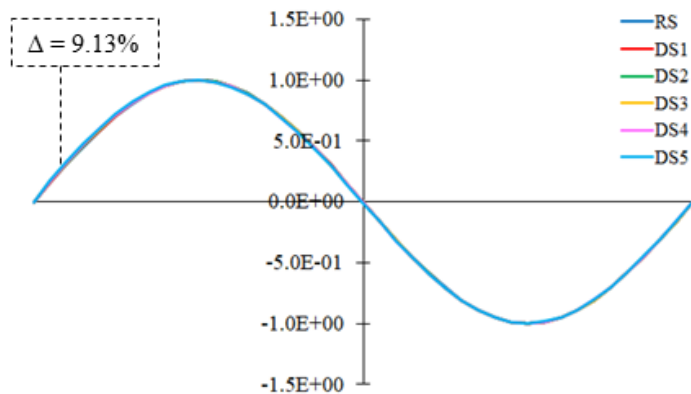

$3^{\text {rd }}$ mode: real part

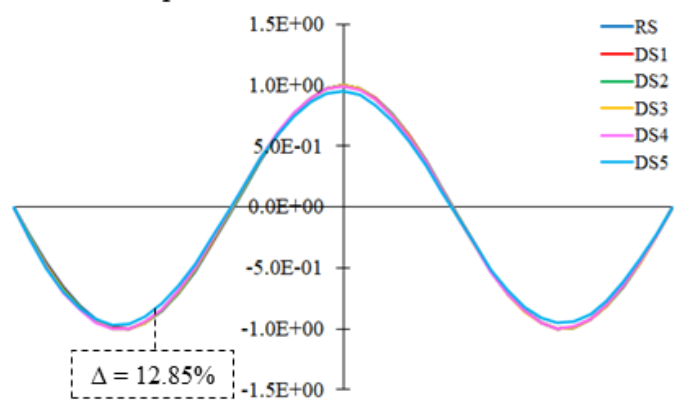

$4^{\text {th }}$ mode: real part

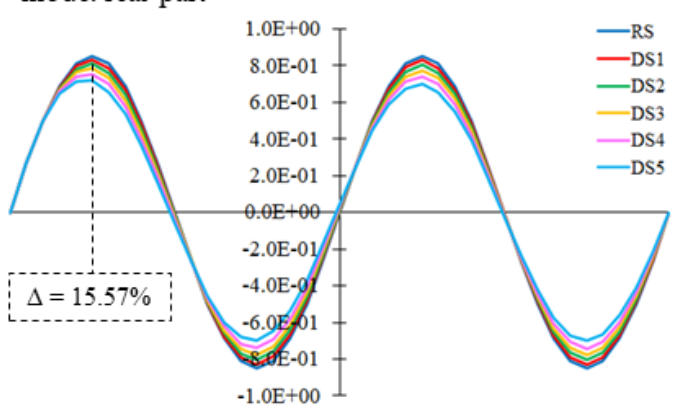

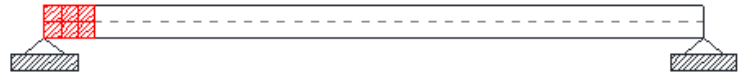

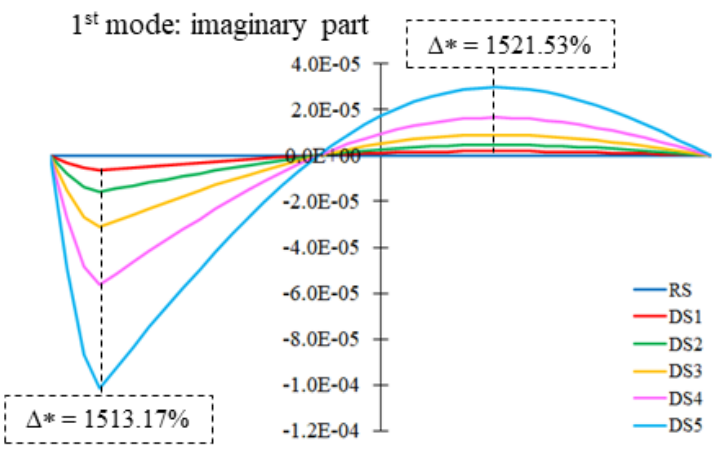

$2^{\text {nd }}$ mode: imaginary part

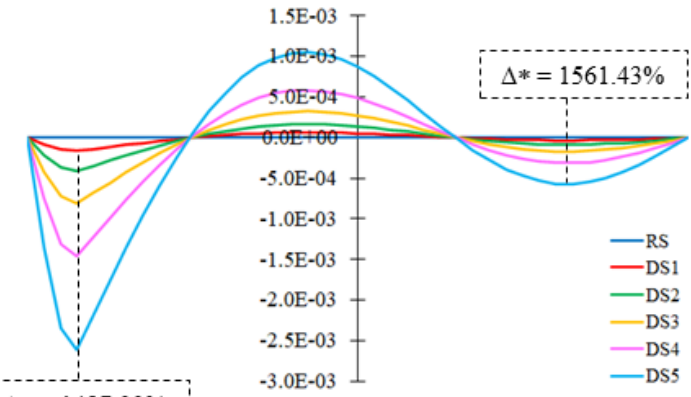

$\Delta^{*}=1487.00 \%$

$3^{\text {rd }}$ mode: imaginary part

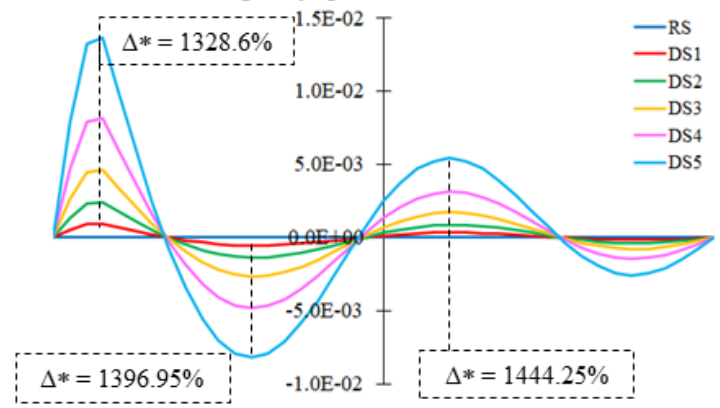

$4^{\text {th }}$ mode: imaginary part

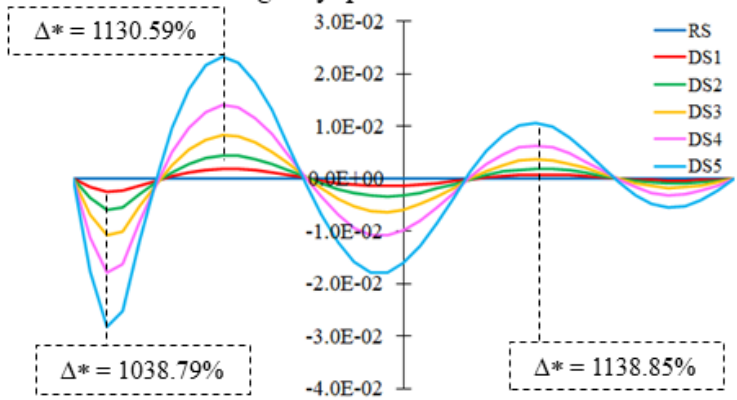

Figure 8. Case b): real component (on the left), and imaginary component (on the right) of the four mode shapes, across damage scenarios. (For the interpretation of colours in the figure legend, the reader is referred to the web version of this article). 


\subsection{Damped arch under progressive damage scenarios and multiple damage locations}

The second numerical example concerned the arch structure depicted in Figure 1. The system was fully clamped at the springings and its geometry featured a mean radius of $1.22 \mathrm{~m}$, a span of $1.90 \mathrm{~m}$, a cross-section of $0.075 \times 0.43 \mathrm{~m}^{2}$ (radial thickness $\times$ arch width) and a springing angle of about $39^{\circ}$. Forced to move in the $x$-z plane, the arch was discretized into 26 straight beam elements (element no. 9 of the NOSA-ITACA library [39]) for a total of 27 nodes and 162 degrees of freedom. Although comparable with the beam structure from a modelling point of view, the example of the arch was deemed necessary to extend the conclusions drawn for beam-like structures to catenary-shaped structural elements featuring different support conditions. In accordance with the previous case, a preliminary modal analysis was conducted in the NOSA-ITACA code to estimate the natural frequencies and mode shapes of the arch in the reference scenario (RS), keeping the same assumptions made for the beam in terms of material properties: elastic modulus $E=3.00 \mathrm{GPa}$, Poisson's ratio $v=0.2$ and mass density $\rho=1800 \mathrm{~kg} / \mathrm{m}^{3}$. The natural frequencies estimated for the first four vibration modes are reported in Figure 9 together with the corresponding mode shapes. Dealing with a 2D problem, modal displacements are all contained in the $x$-z plane and follow the typical sequential configuration expected for segmental arches on fixed supports: a first asymmetric mode in longitudinal direction, two symmetric bending modes in vertical direction and an additional asymmetric bending mode in vertical direction. Note that the number of inflection points, thus curvatures, progressively increases for higher-order frequencies.

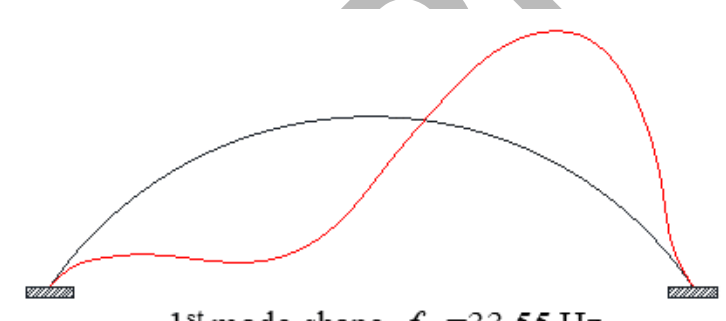

$1^{\text {st }}$ mode shape, $f_{1 \mathrm{i}}=33.55 \mathrm{~Hz}$

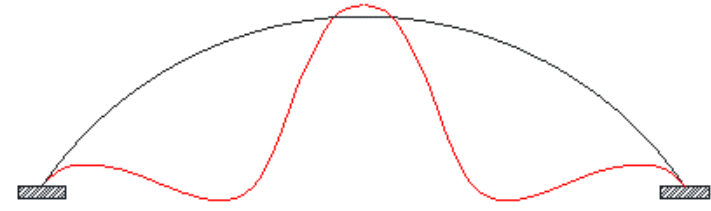

$3^{\text {rd }}$ mode shape, $f_{3 \mathrm{i}}=101.41 \mathrm{~Hz}$

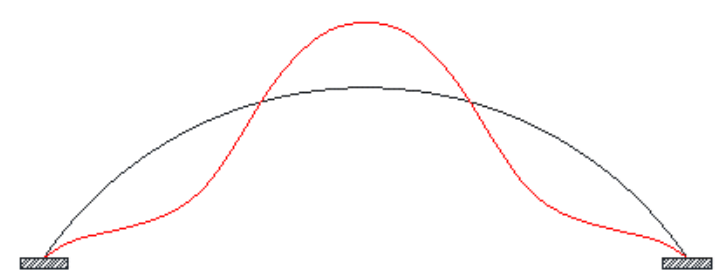

$2^{\text {nd }}$ mode shape, $f_{2 \mathrm{i}}=59.95 \mathrm{~Hz}$

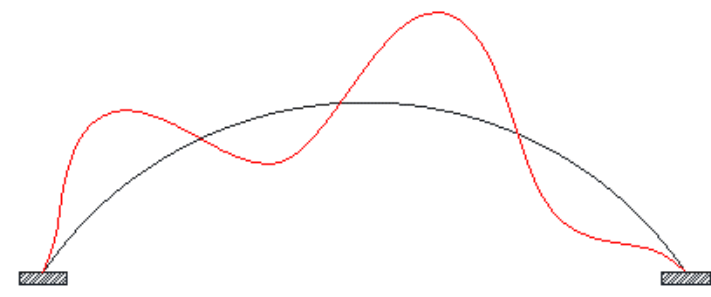

$4^{\text {th }}$ mode shape, $f_{4 \mathrm{i}}=116.01 \mathrm{~Hz}$

Figure 9. The first four vibration modes of the arch in the reference scenario 
The damping matrix $\mathrm{C}$ of the undamaged state was calculated as a linear combination of the mass $\mathrm{M}$ and stiffness $\mathrm{K}$ matrices according to the expressions given in Eqs. (1.20) and (1.21), and assuming a damping ratio equal to $3 \%$. Then, five damage scenarios (DS1-DS5) were simulated by progressively reducing the elastic modulus of a few elements in multiple locations, so as to lead to the development of three plastic hinges $c_{1}, c_{2}$ and $c_{3}$ in notorious positions (Table 1).

Table 1. Reduction of the elastic modulus over damage scenarios

\begin{tabular}{lllll}
\hline Scenario & $C_{1}$ & $C_{2}$ & $C_{3}$ & Crack location \\
\hline DS1 & -- & $0.90 E$ & -- \\
DS2 & $0.95 E$ & $0.80 E$ & -- \\
DS3 & $0.90 E$ & $0.70 E$ & $0.95 E$ \\
DS4 & $0.80 E$ & $0.60 E$ & $0.90 E$ \\
DS5 & $0.70 E$ & $0.50 E$ & $0.85 E$ \\
\hline
\end{tabular}

The outcome of the complex modal analyses is summarized in Figure 10 and Figure 11 in terms of frequency decay, MAC coefficients and modal complexity indices over the different DSs. The close inspection of the graphs reveals a monotonic decrease of both longitudinal and vertical bending stiffness, reading relative frequency downshifts up to $6 \%$ in the last scenario (DS5); sensible variations are also noticed in the deflection characteristics of the arch related to the vertical bending modes, which indeed result the most affected by the three-hinge damage configuration. As regards the modal complexity indices, the results are in line with what observed for the beam cases: apart from the MPD index, all the other indices share the same trend and prove to be sensitive to the mode shape complexity originated by multiple and progressive damage scenarios, showing a monotonic increase with the damage evolution particularly strong for higher-frequency modes. Notwithstanding, no useful information about the spatial location of the cracks is retrieved through these scalar indicators.

With the aim of moving to a higher level of damage identification, the evolution of the real and imaginary parts of the most representative mode shapes of the arch are plotted and analysed along $x$ and $z$ directions for the different damage scenarios: mode $\phi_{1}$ that is the fundamental mode of the structure and the only one with prevalent modal components in longitudinal 
direction; and mode $\phi_{4}$ that is the only mode with dominant vertical modal components showing the lowest MAC value and therefore the greatest damage-induced deflection changes.
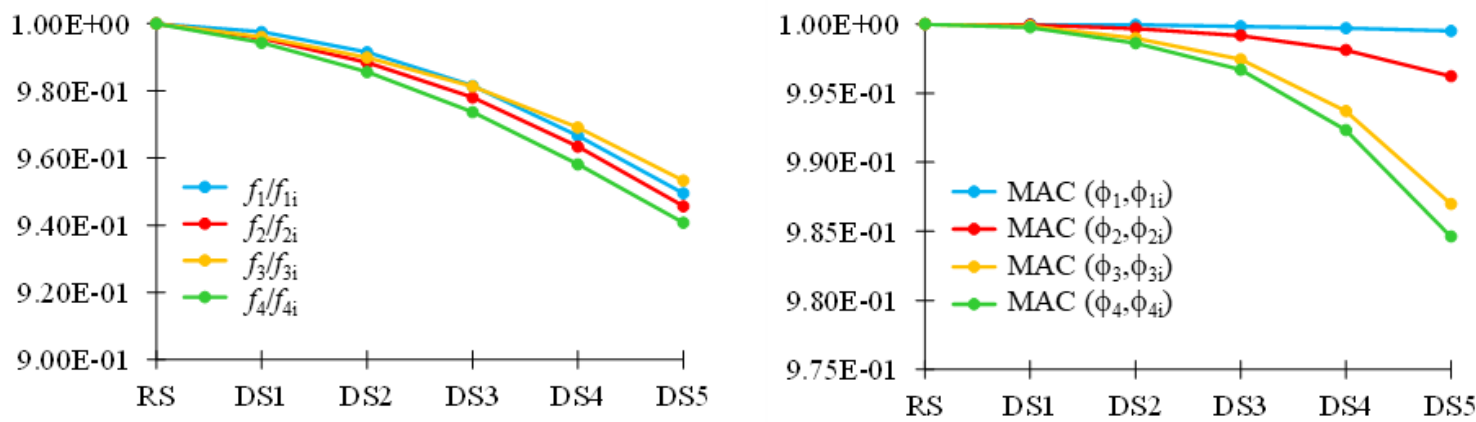

Figure 10. Case c): frequency decay and MAC trend versus damage scenario (DS). (For the interpretation of colours in the figure legend, the reader is referred to the web version of this article).
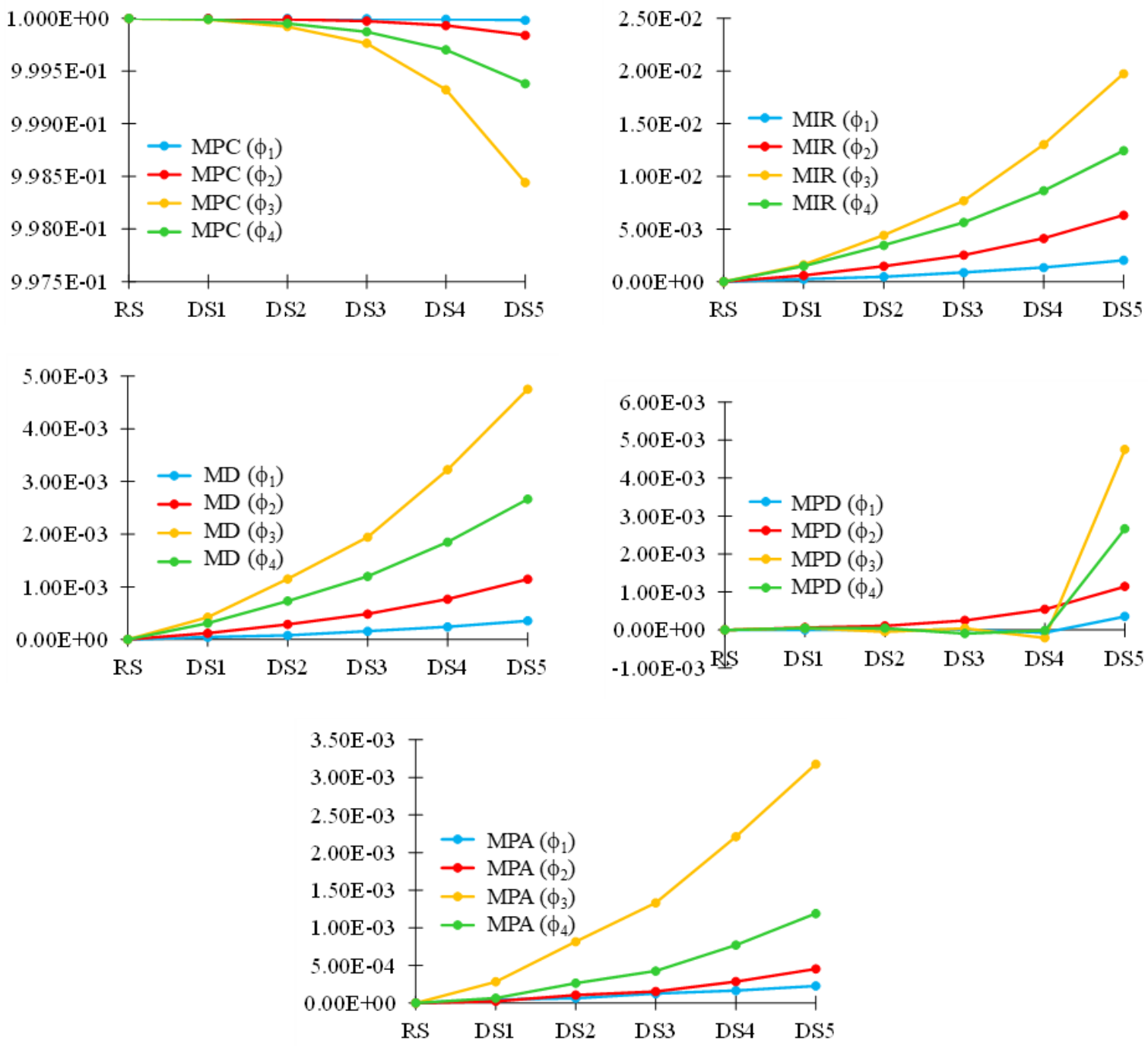

Figure 11. Case b): MPC, MIR, MD, MPD and MPA trend versus damage scenarios (DS). (For the interpretation of colours in the figure legend, the reader is referred to the web version of this article). 


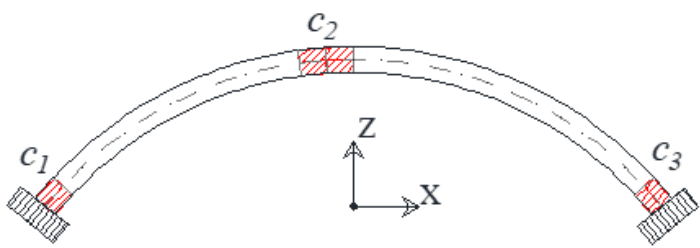

$1^{\text {st }}$ mode: real part ( $\mathrm{x}$ component)

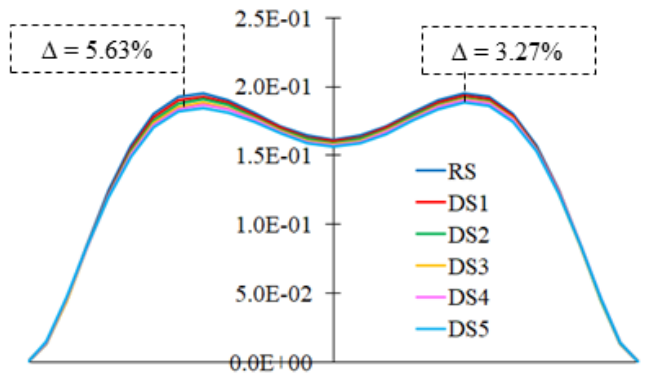

$1^{\text {st }}$ mode: imaginary part ( $\mathrm{x}$ component)

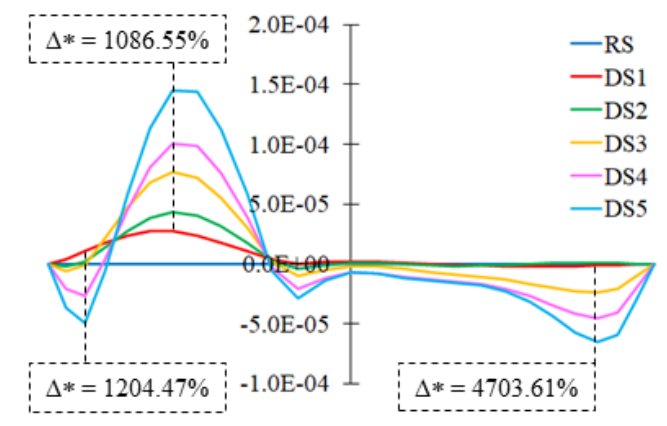

$4^{\text {th }}$ mode: real part ( $x$ component)

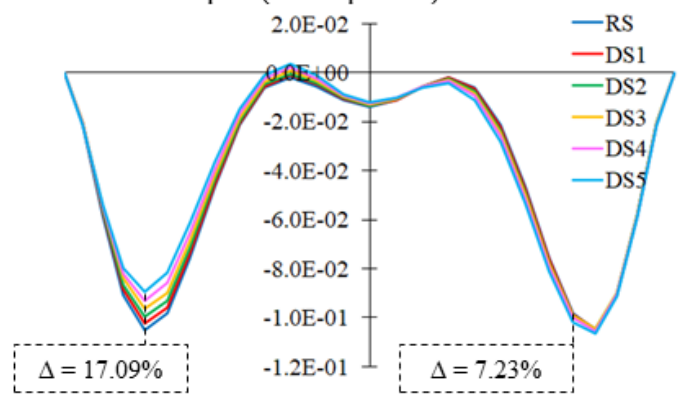

$4^{\text {th }}$ mode: imaginary part ( $\mathrm{x}$ component)

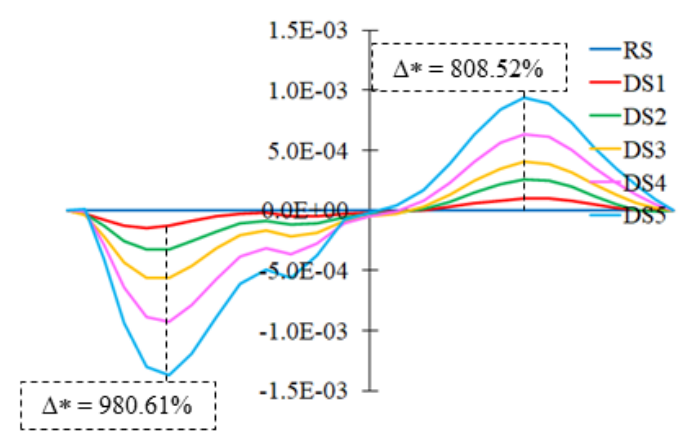

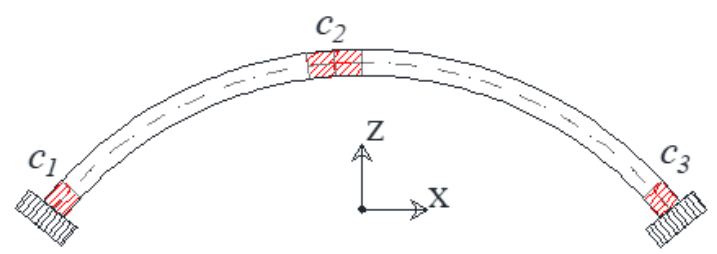

$1^{\text {st }}$ mode: real part ( $\mathrm{z}$ component)

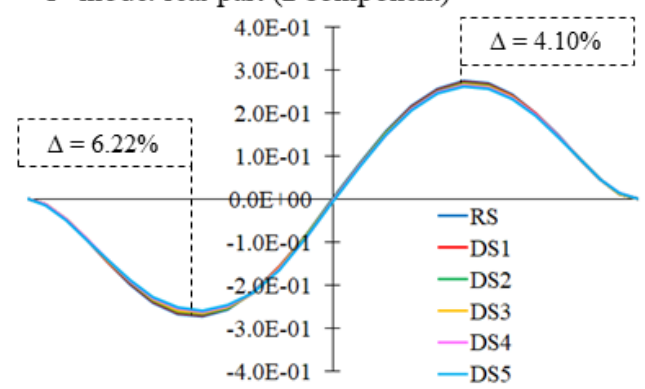

$1^{\text {st }}$ mode: imaginary part ( $\mathrm{z}$ component $)$

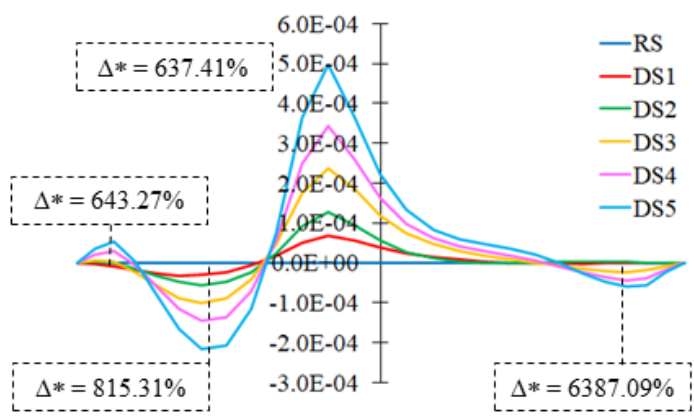

$4^{\text {th }}$ mode: real part ( $z$ component)

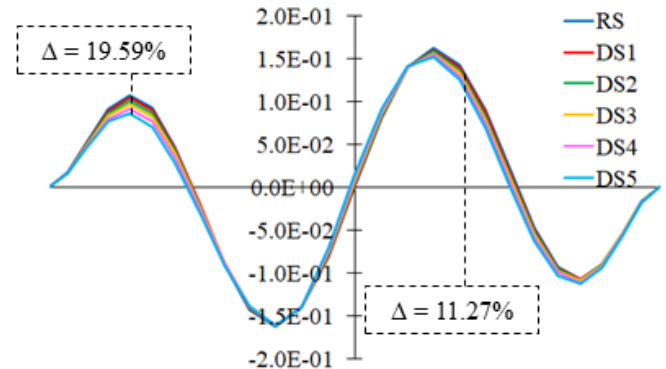

$4^{\text {th }}$ mode: imaginary part (z component)

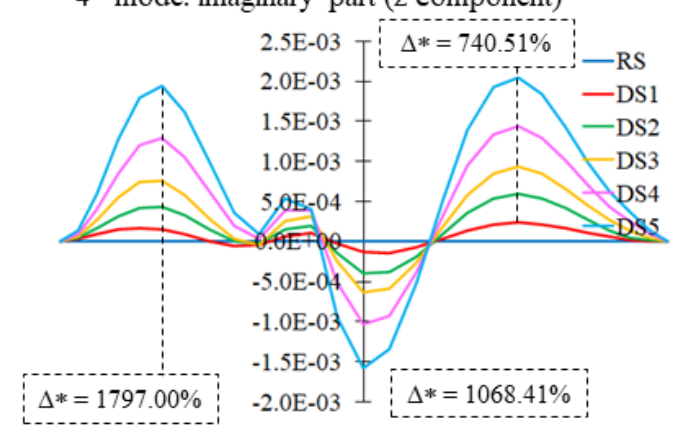

Figure 12. Case c): real (upper) and imaginary (lower) components variation of the first and fourth mode shapes of the arch with progressive and multiple damage scenarios. (For the interpretation of colours in the figure legend, the reader is referred to the web version of this article). 
As shown in Figure 12, the imaginary components along $x$ and $z$ directions are very sensitive to damage unlike their real counterparts and the peak values in terms of percentage variation are reached nearby the areas where the three plastic hinges $c_{1}, c_{2}$ and $c_{3}$ were formed. Relatively to the first mode shape, the highest percentage variations of the imaginary part along $x$ direction are attained close to the arch supports, a phenomenon in line with the fact that the hinges occurring at the ends of catenary-shaped structures greatly influence the modes with dominant in-plane longitudinal components; vice versa, the major percentage variation of the imaginary components along the $z$ direction is reached in correspondence of the central hinge. Regarding the imaginary content of the fourth mode shape, the greatest percentage variations are recorded nearby all three affected areas for the $z$ direction, whereas only the extremity components of the mode feature major changes in $x$ direction. This was somehow expected, given the major impact of apex damages on the vertical bending response of arch structures.

\section{Analysis of modal complexity using experimental data}

As pointed out in the Introduction, during past three decades several authors have tried to analyse and quantify the complexity of vibration modes in dynamic systems for damage identification purposes. To the best knowledge of the authors, except for rare instances [14], most of the practical examples that were used to validate the aforementioned theorical formulations and findings relied on simple linear discrete models featuring diagonally lumped mass matrices, symmetric and banded stiffness matrices, and controllable proportional or nonproportional damping matrices. With such assumptions, as demonstrated in Section 3 for analogous cases, all the candidate modal complexity indices retrieved from the literature result effective for detecting the presence of structural damage, where this implies a loss of stiffness and an increase of dissipation - with respect to the initial undamaged configuration - that lead to a non-proportionally damped system characterized by complex mode shapes. Yet, none of these indices provide spatial indications about the damage location, thereby adding no extra value to the conventional and straightforward damage identification procedures based on the comparison of frequency values, likewise (if not more) sensitive to damage scenarios induced by stiffness losses.

The complexity of the problem further increases when dealing with real-world physical systems, whose estimated mode shapes are not exactly real-valued monophase vectors even for undamaged conditions, and the source of complexity is not solely due to nonproportional 
damping. Hence the need to benchmark numerical and analytical studies with verified experimental data in order to fully validate their effectiveness.

The laboratory application described hereafter is examined with the aim of showing the underlying challenges associated to real experimental test cases and the difficulties that arise in identifying the damage when state-of-the-art formulations based on modal complexity are applied. In order to overcome the limitations that literature indices exhibit for experimental use, a new pointwise indicator relying on the imaginary content of measured complex modes is proposed for both localization and assessment of structural damage.

\subsection{Description of the experimental benchmark}

Benchmark of the present study is the experimental campaign carried out on a reduced-scale arch specimen tested by the authors in the structural laboratory of the University of Minho (Guimarães, Portugal) [40]. The arch was built with low compressive brick units of dimensions $100 \times 50 \times 75 \mathrm{~mm}^{3}$ (length $\times$ width $\times$ height) laid lengthwise over the arch width with a running bond pattern and assembled using low-quality lime mortar joints of less than $10 \mathrm{~mm}$ thickness. The structure was erected on two concrete abutments, one fixed to the floor by bolts and the other one resting on a simple supporting system with lateral guide rollers to allow for horizontal sliding. Aimed at tracking the dynamic behaviour of this type of structures when subjected to one-sided horizontal settlements, the arch was progressively damaged by imposing outward increasing displacements at the movable support, reaching five unrecoverable Damage Scenarios (DSs). Mass loading effects were also considered by placing on the arch spandrels two lime bags of $25 \mathrm{~kg}$ each (corresponding to about 50\% of the structure's weight) before proceeding with the static tests.

After each displacement stage, dynamic identification tests were performed to fully characterize the modal response of the system and to follow its stiffness degradation with increasing structural damage. A dense network of high-sensitivity wired accelerometers was deployed for the purpose, enabling to obtain a well distributed measurement array along the back and front edges of the arch, in both tangential and normal direction, for a total of 52 nodal processes (13 measurement points per edge). Such a distribution was deemed good enough to attain a fine resolution in terms of mode shapes, which is crucial to identify the position of localized damages. Figure 13 illustrates the geometry of the scaled arch together with the static and dynamic test layouts. 

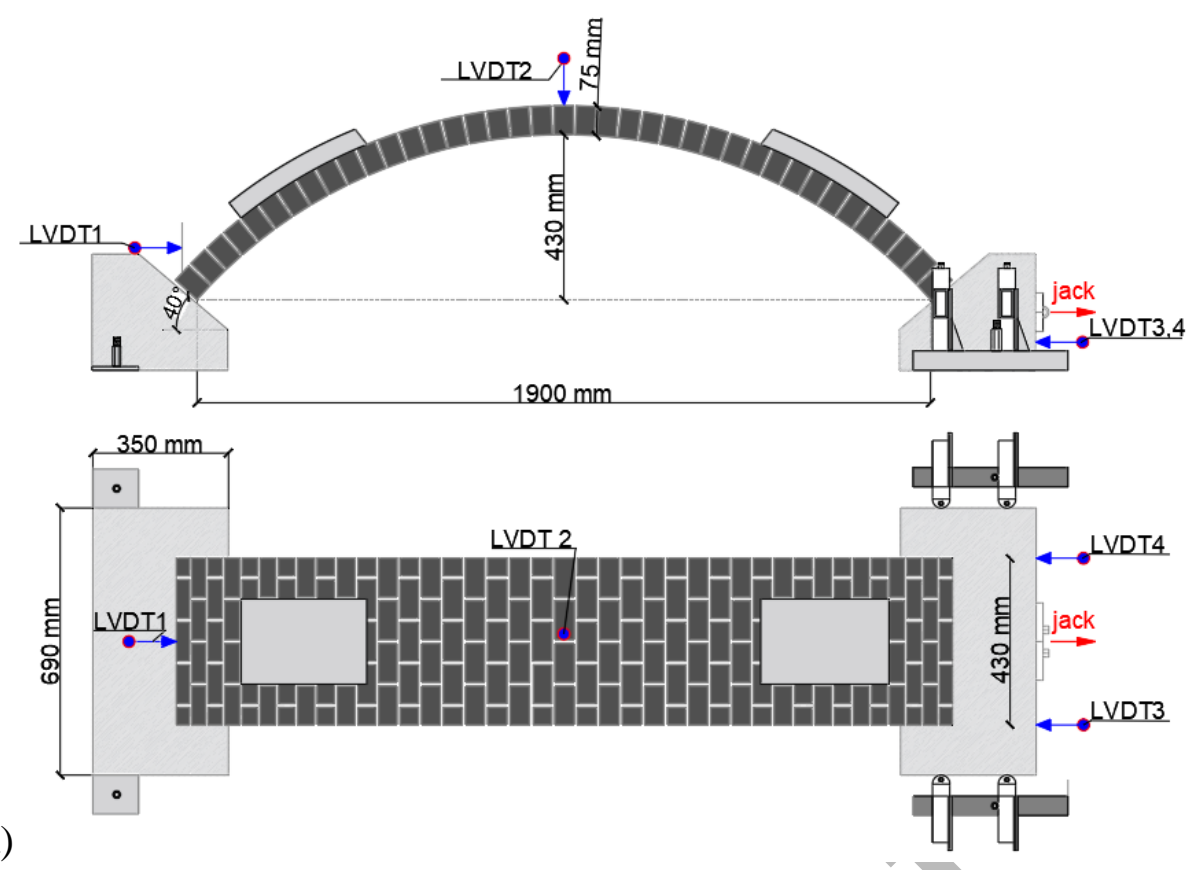

a)

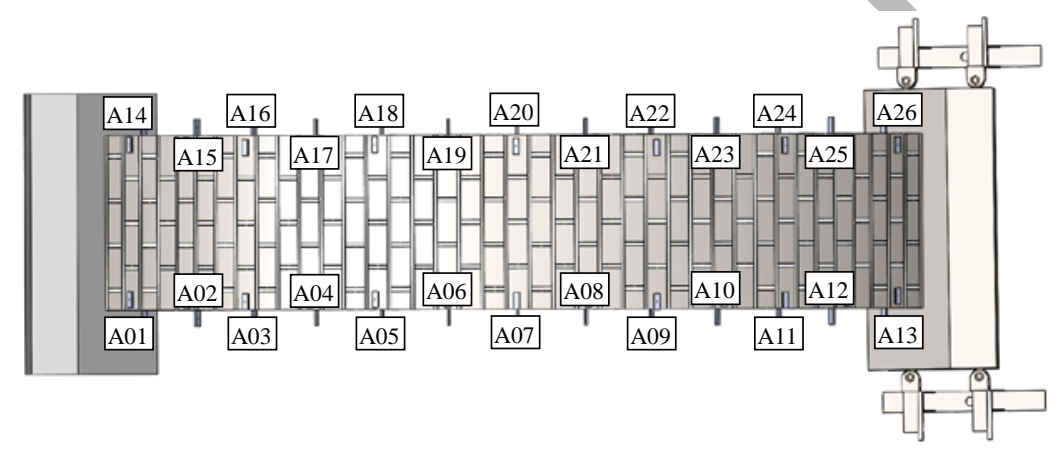

b)

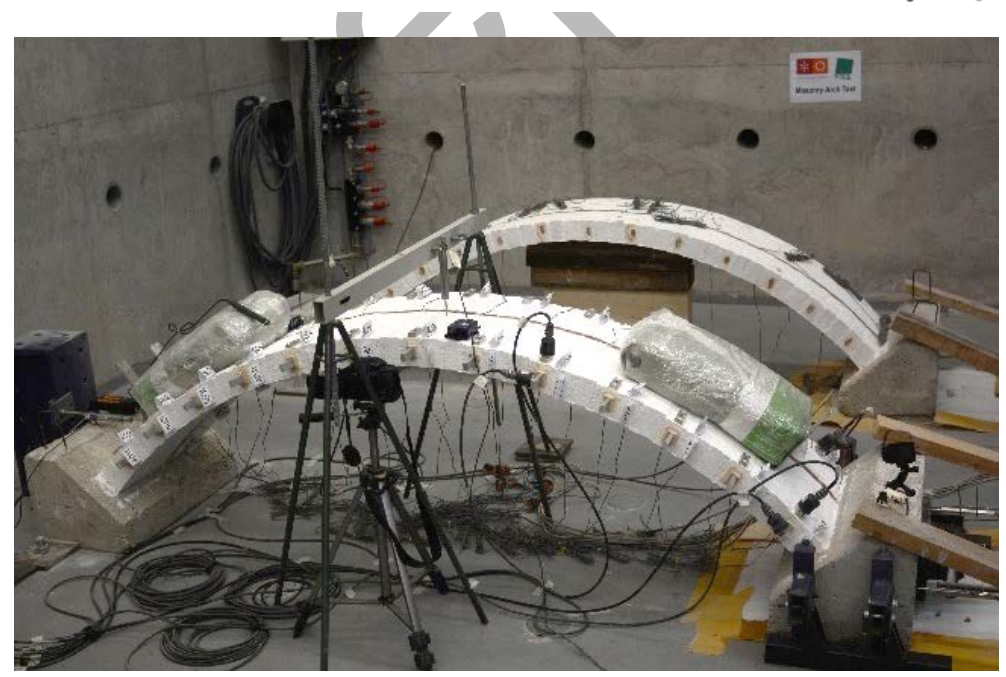

Figure 13. Geometry of the experimental arch along with the (a) static and (b)-(c) dynamic test layouts.

Minimum durations of $180 \mathrm{~s}$ and $60 \mathrm{~s}$ were fixed for ambient and random impact vibration tests, respectively, so as to ensure a time window greater than 2000 times the fundamental period of the arch (the second source of excitation was necessary to improve the low signal-to- 
noise ratio of the outputs due to the non-negligible background noise of the laboratory). To eliminate possible aliasing effects during post-processing operations, signals were acquired with a sampling rate of $400 \mathrm{~Hz}$, which corresponded to twice the highest frequency of interest for the undamaged structure (i.e. the Nyquist frequency). Twelve setups, each one consisting of four reference sensors and four moving sensors, were necessary to record the nodal response processes of all pre-established measurement points.

While the movable support was displaced through a hydraulic jack, an asymmetric crack pattern progressively developed at the mortar-brick interfaces leading to the onset of three hinges distributed according to the classical "intrados-extrados-intrados" configuration expected for segmental arches on spreading supports. The first crack $c_{1}$ occurred at the intrados, in the left region of the keystone, close to sensor A05; whereas, the second and the third cracks, $c_{2}$ and $c_{3}$, appeared at the extrados of the skewbacks corresponding to the fixed and moving supports, close to sensors A01 and A13, respectively. The crack pattern observed at the end of the five stages ( $3 \mathrm{~mm}$ settlement) is presented in Figure 14. For a complete description of the experimental campaign and a thorough analysis of the arch response up to failure, the reader is referred to [40].
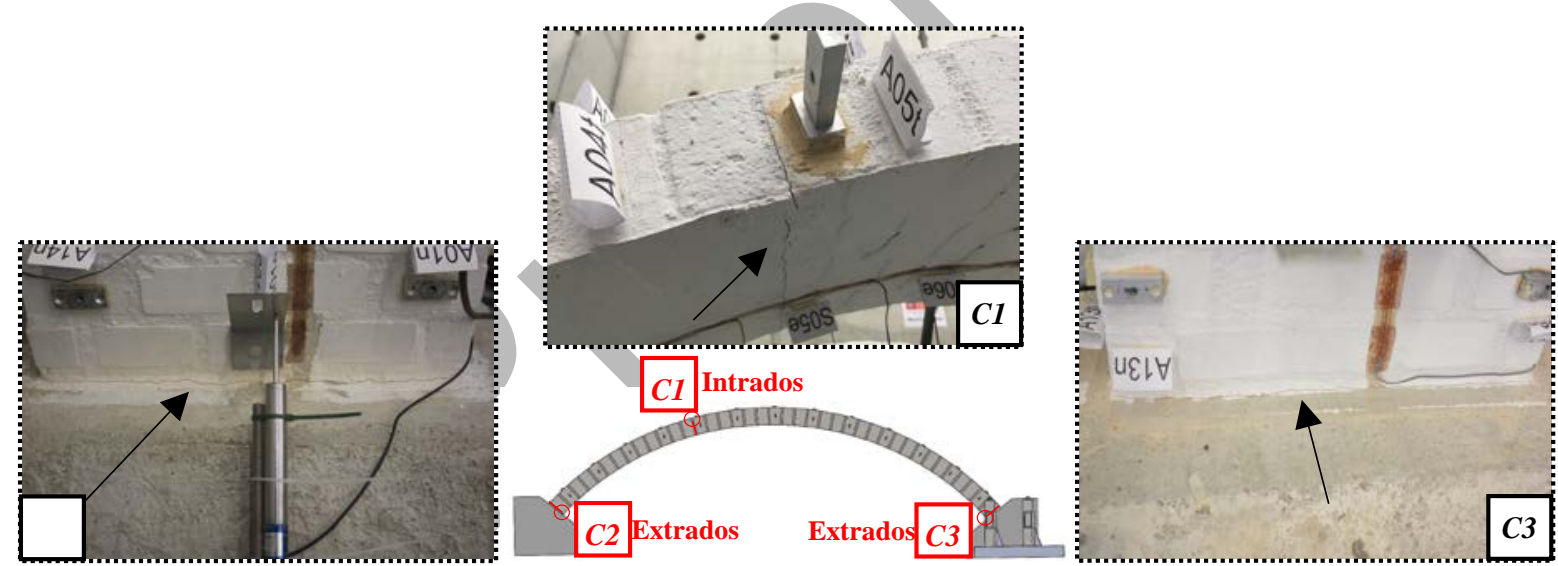

Figure 14. Crack pattern of the masonry arch after five displacement stages (3 mm settlement).

\subsection{Operational modal analysis and effects of crack location}

The extraction of the experimental modal parameters over the five DSs was carried out by cross-validating the estimates from two well-known and established OMA (Operational Modal Analysis) techniques implemented in the software ARTeMIS Modal: the Enhanced Frequency Domain Decomposition (EFDD) and the Stochastic Subspace Identification with Extended Unweighted Principal Component (SSI-UPCX). In the following, for the sake of brevity, only the modal features extracted by the SSI-UPCX will be considered. 
Table 2 and Table 3 summarize the results obtained in terms of mean frequency values $(f)$ and damping ratios $(\xi)$ across the different scenarios. For completeness, a visual insight into the mode shapes configuration of the reference scenario (RSW) versus the last damage scenario (DS5) is provided in Figure 15, where the MAC values between corresponding mode pairs are also displayed. Overall, six to seven vibration modes in the frequency range 30-150 Hz were identified and tracked with progressive damage [40]: three in-plane vibration modes, one in longitudinal direction $\mathrm{X}\left(\phi_{1}\right)$ and three in vertical direction Z (of which two symmetric bending modes $\phi_{3}-\phi_{6}$, and one asymmetric double bending mode $\phi_{4}$ ), and three out-of-plane vibration modes $\left(\phi_{2}, \phi_{5}\right.$ and $\left.\phi_{7}\right)$.

As the support slid outwards, the arch cracked and this sensibly degraded the structural stiffness, leading to frequency drops up to $36 \%$ and $22 \%$ for modes $\phi_{1}$ and $\phi_{4}$. Nevertheless, for the same modes, the MAC coefficients between $\mathrm{RS}_{\mathrm{W}}$ and DS5 modal vectors are equal to 0.98 and 0.60 respectively, meaning that the largest differences in the modal displacements imputable to damage only concerned mode $\phi_{4}$. This outcome is in accordance with the behaviour expected for this type of damage pattern; indeed, given the prevalence of horizontal modal displacement components, the presence of crack hinges at the supports does not influence the dominant vibration mode but in terms of longitudinal stiffness, while the presence of the crack on the left region of the keystone does remarkably affect both the bending vertical stiffness of the structure and the associated mode shape with higher vertical components. Based on these premises, the damage analysis detailed in sub-Sections 4.3 and 4.4 will focus exclusively on the in-plane modes of the experimental arch.

Table 2. Variation of the natural frequencies of the arch with progressive damage.

\begin{tabular}{ccccccccc}
\hline \multirow{2}{*}{ Mode } & \multicolumn{7}{c}{ Frequencies [Hz] } \\
\cline { 2 - 9 } & RS & RS & DS1 & DS2 & DS3 & DS4 & DS5 & $\Delta f^{*}[\%]$ \\
\hline 1 & 37.03 & 30.06 & 26.31 & 23.40 & 21.44 & 20.20 & 19.16 & -36.26 \\
2 & 58.64 & 50.95 & 50.28 & 48.86 & 45.14 & 44.05 & 43.19 & -15.23 \\
3 & 63.56 & 59.44 & 59.04 & 58.14 & 58.27 & 57.77 & 57.54 & -3.20 \\
4 & 100.76 & 95.23 & 80.47 & 75.75 & 74.97 & 74.39 & 74.09 & -22.20 \\
5 & 122.67 & 120.62 & 113.16 & 111.44 & 110.60 & 110.98 & 110.86 & -8.09 \\
6 & 125.06 & - & - & 121.50 & 118.71 & 116.68 & 115.78 & -7.42 \\
7 & 146.21 & 134.02 & 136.40 & 130.91 & 128.84 & 129.60 & 129.00 & -3.75 \\
\hline
\end{tabular}

*Percentage variation between reference (RSw) and last damage (DS5) scenarios. 
Table 3. Variation of the damping ratios of the arch with progressive damage.

\begin{tabular}{ccccccccc}
\hline \multirow{2}{*}{ Mode } & \multicolumn{7}{c}{ Damping ratios [\%] } \\
\cline { 2 - 9 } & RS & RS & DS1 & DS2 & DS3 & DS4 & DS5 & $\Delta \xi *[\%]$ \\
\hline 1 & 0.95 & 1.26 & 2.27 & 2.99 & 2.90 & 2.59 & 2.14 & +69.84 \\
2 & 0.88 & 3.06 & 1.39 & 3.79 & 4.62 & 3.75 & 1.84 & -39.87 \\
3 & 1.02 & 1.22 & 3.61 & 1.86 & 1.83 & 1.77 & 2.02 & +65.57 \\
4 & 0.97 & 2.12 & 3.27 & 2.61 & 2.39 & 1.98 & 2.09 & -1.42 \\
5 & 1.25 & 2.97 & 1.57 & 2.95 & 2.99 & 3.03 & 2.69 & -9.43 \\
6 & 1.03 & - & - & 3.86 & 3.17 & 3.21 & 2.49 & +141.75 \\
7 & 1.46 & 4.19 & 1.79 & 2.23 & 2.00 & 2.58 & 3.46 & -17.42 \\
Avg & 1.08 & 2.47 & 2.32 & 2.90 & 2.84 & 2.70 & 2.39 & +29.86 \\
\hline
\end{tabular}

*Percentage variation between reference (RSw) and last damage (DS5) scenarios.

Lastly, it is worth drawing attention to the variation of energy dissipation among the different scenarios. As a rule, the vibration modes of a damaged structure feature higher damping values than their undamaged counterparts. Accordingly, for the present arch, one would expect a progressive increase of modal damping between subsequent scenarios consistent with the decay of the system oscillations after the damage onset, but this is not the case even though an overall damping increase of about $30 \%$ is recorded with respect to the reference configuration if the contribution of all modes is considered. Such a behaviour clearly reveals the complex nature of this modal parameter, whose accurate identification presents much higher statistical variation from random error sources than modal frequencies, thereby explaining why the damping ratio is rarely adopted for structural health monitoring purposes. The simultaneous occurrence of multiple sources of complexity beyond the damage can strongly affect the energy dissipation mechanisms of physical systems and this is particularly evident for the structure under consideration, where higher modal complexity factors $(\mathrm{MCF}=1-\mathrm{MPC})$ do not correspond to increased damage, as shown in Figure 16 (mode $\phi_{6}$ was not identified neither in $\mathrm{RS}_{\mathrm{W}}$ nor in DS1). 


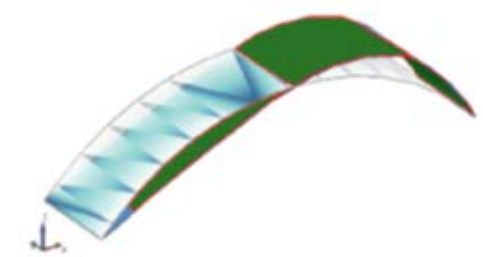

Mode $1(\mathrm{MAC}=1.00)$

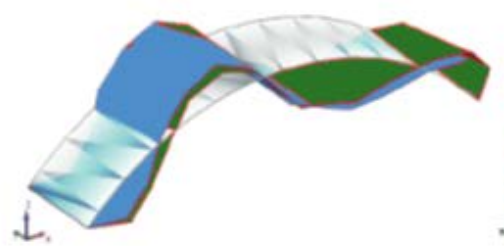

Mode $4(\mathrm{MAC}=0.90)$

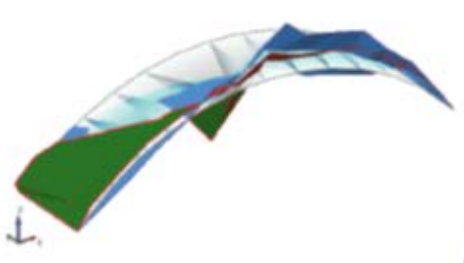

Mode $2($ MAC $=0.70)$

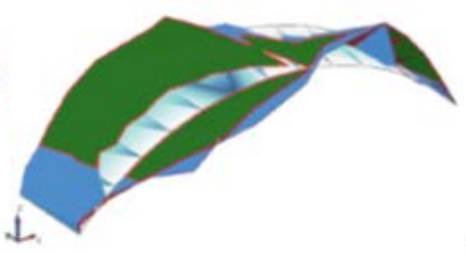

Mode 5 (MAC $=0.71)$

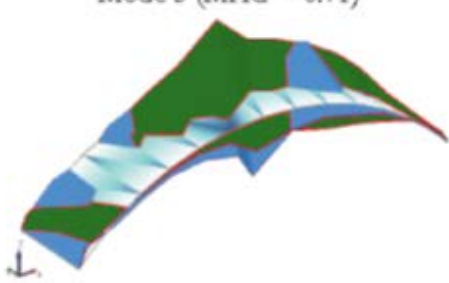

Mode 7 (MAC $=0.67)$

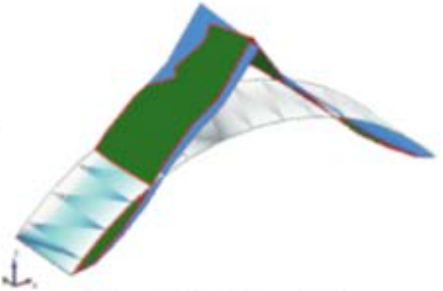

Mode $3(\mathrm{MAC}=0.93)$

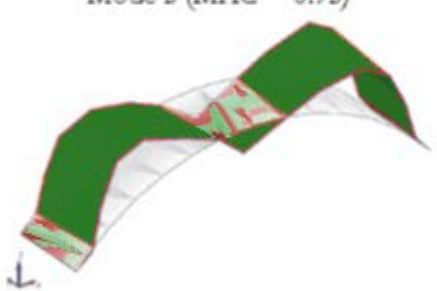

Mode 6

Figure 15. Mode shapes comparison between reference and last damaged scenarios (undeformed shape in grey, $\mathrm{RS}_{\mathrm{w}}$ in blue and DS5 in green).

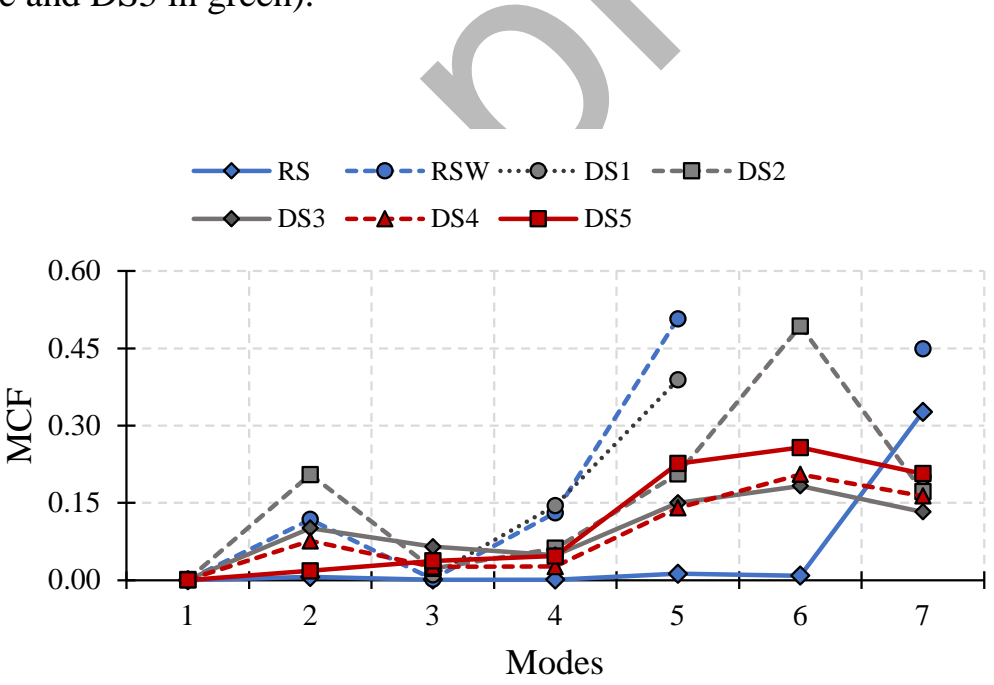

Figure 16. MFC trend of the seven mode shapes estimated along the DSs. (For the interpretation of colours in the figure legend, the reader is referred to the web version of this article).

\subsection{Normalization and rotation of identified complex modes for experimental use}

As stated in the previous sections, for non-proportionally damped systems the mode shapes are in general complex, but part of this complexity is dummy and must be removed to exploit the magnitude of their complex nature as a measure of structural damage. To this end, the arch 
modes experimentally identified were normalized and rotated according to the procedure recalled in the Section 2.2 so as to minimize their imaginary part and maximize the real one [3].

Figure 17 and Figure 18 display the polar plots of the arch eigenmodes for the reference scenarios (RS and $\mathrm{RS}_{\mathrm{W}}$ ) before and after removing the fictitious complexity. Note that both horizontal ( $x$ direction - blue colour) and vertical ( $z$ direction - red colour) modal components are represented onto the complex plane. It is observed that, after the normalization and rotation, all modal vectors estimated for RS (with the exception of mode $\phi_{7}$ ) become real and coincide with the real ones that would be obtained for zero damping, meaning that the system in its initial reference configuration is proportionally damped. Conversely, by replicating the same operation for $\mathrm{RS}_{\mathrm{W}}$, the mode shapes do not turn into real-valued monophase vectors (likely because of the mass loading effects resulting from the addition of two lime bags on the arch spandrels), which means that only part of the mode complexity is fictitious, hence the system is non-proportionally damped even if not yet damaged. This confirms that undamaged structures do not necessarily meet the assumption of proportional damping and that the modal complexity cannot be univocally linked to structural damage.

Interesting considerations also emerge by inspecting the polar plots of the vibration modes of the arch - after normalization and rotation - for two representative damage scenarios, i.e. DS3 and DS5 (Figure 19). Concerning DS5, except for the higher frequency modes (i.e. $\phi_{5}, \phi_{6}$ and $\phi_{7}$ ), all main natural modes of the arch lie almost completely on the real axis, even though a much higher complexity of the in-plane modes would be expected for this last scenario. By contrast, when observing the polar representation of the modal components of the arch for DS3 (less severe than DS5), one can notice that only the fundamental mode of the structure lies completely on the real axis. This outcome corroborates the difficulties in establishing a direct relationship between damage extent and modal complexity in dynamic systems. 
Reference Scenario (RS)

(a)

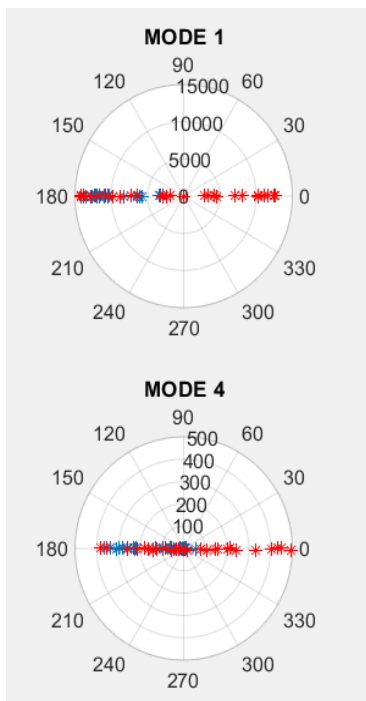

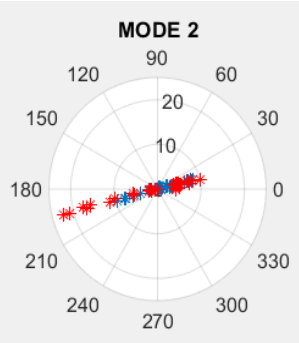

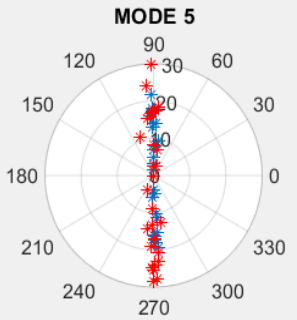

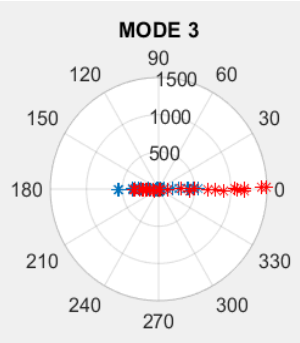
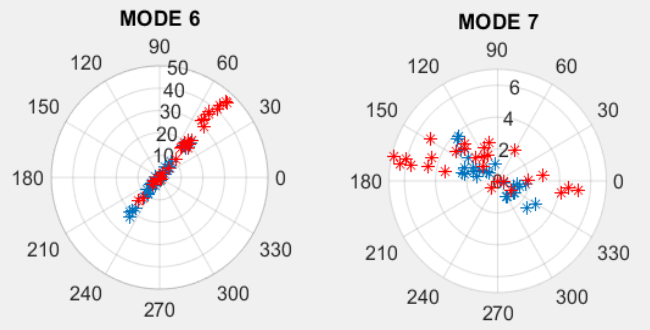

(b)
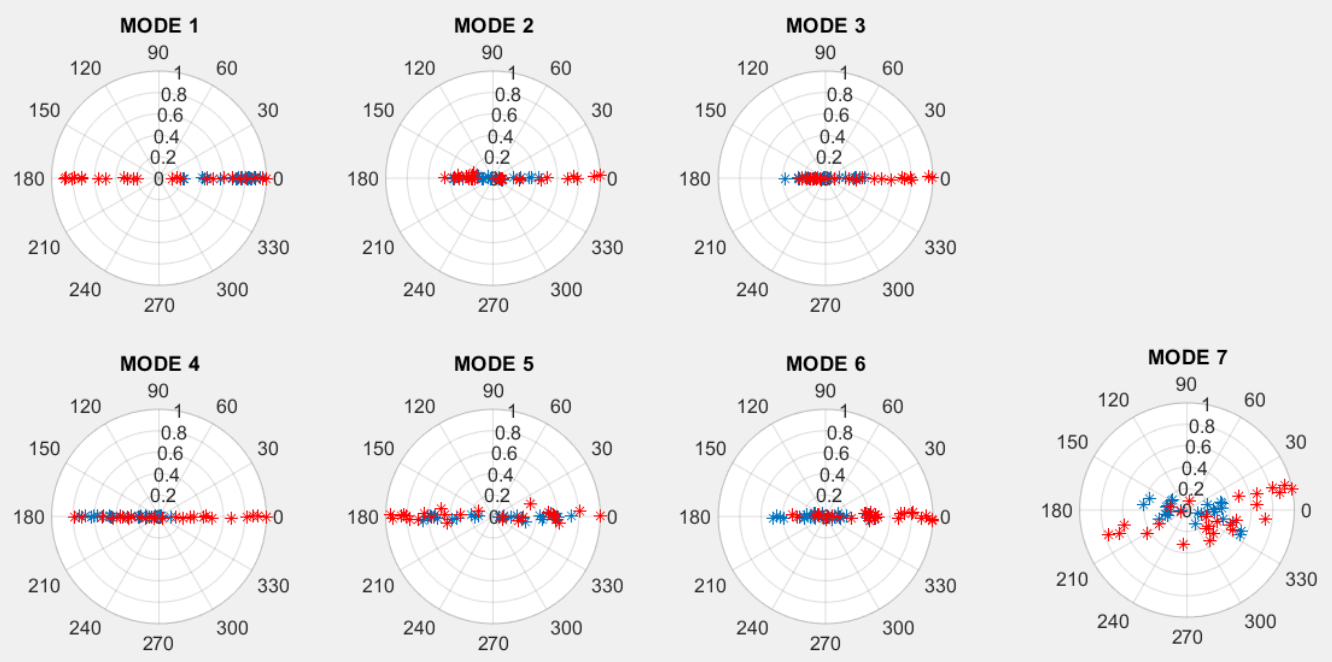

Figure 17. Mode shape components of the arch (a) before and (b) after normalization \& rotation for Reference Scenario RS; $x$ components in blue and $z$ components in red. (For the interpretation of colours in the figure legend, the reader is referred to the web version of this article). 
Reference Scenario with applied load $\left(\mathrm{RS}_{\mathrm{W}}\right)$

(a)

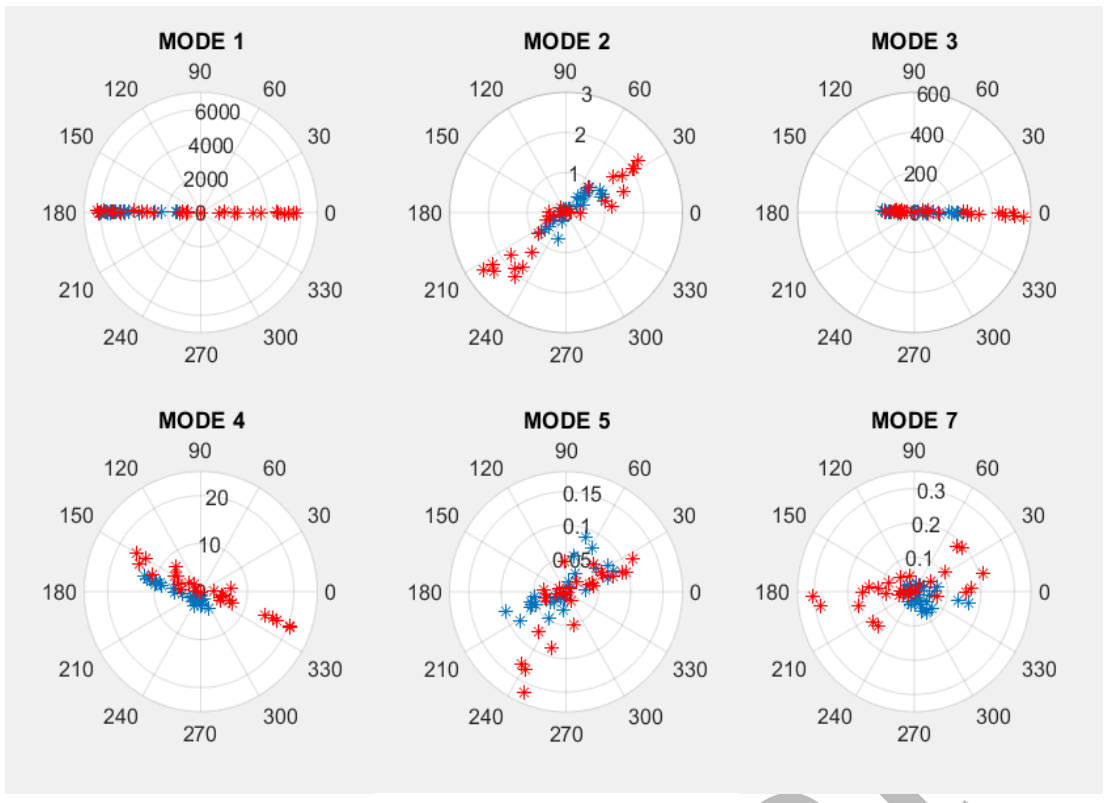

(b)
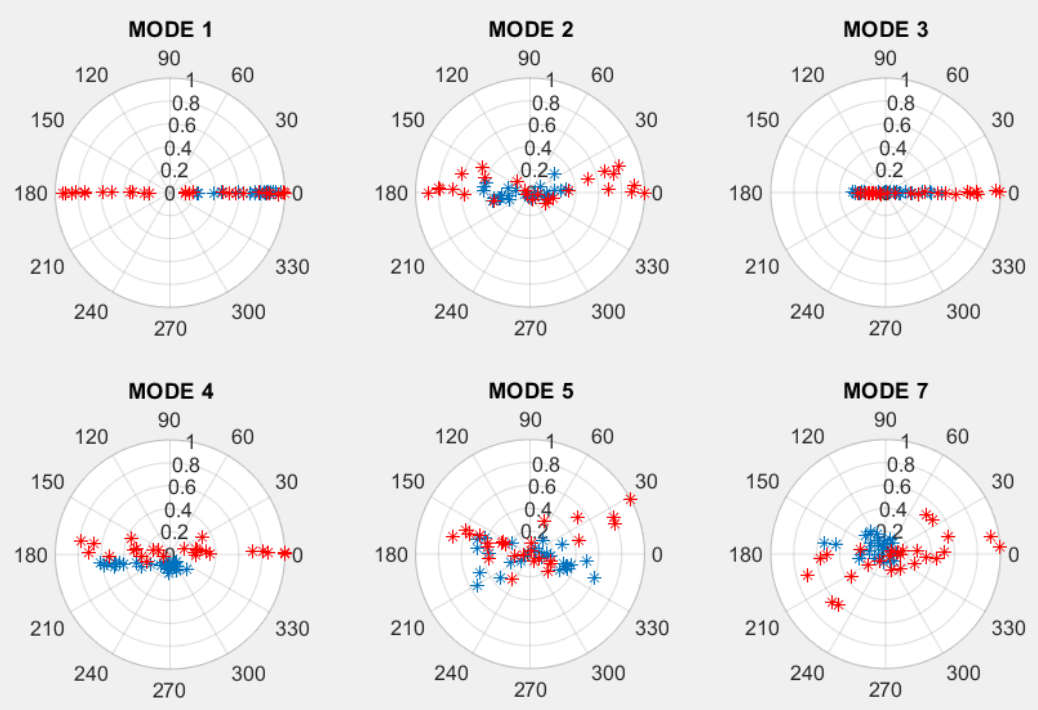

Figure 18. Mode shape components of the arch (a) before and (b) after normalization \& rotation for Reference Scenario RS $\mathrm{w} ; x$ components in blue and $z$ components in red. (For the interpretation of the references to colour in the figure legend, the reader is referred to the web version of this article). 


\section{Damage Scenarios (DS3-DS5)}

(a)

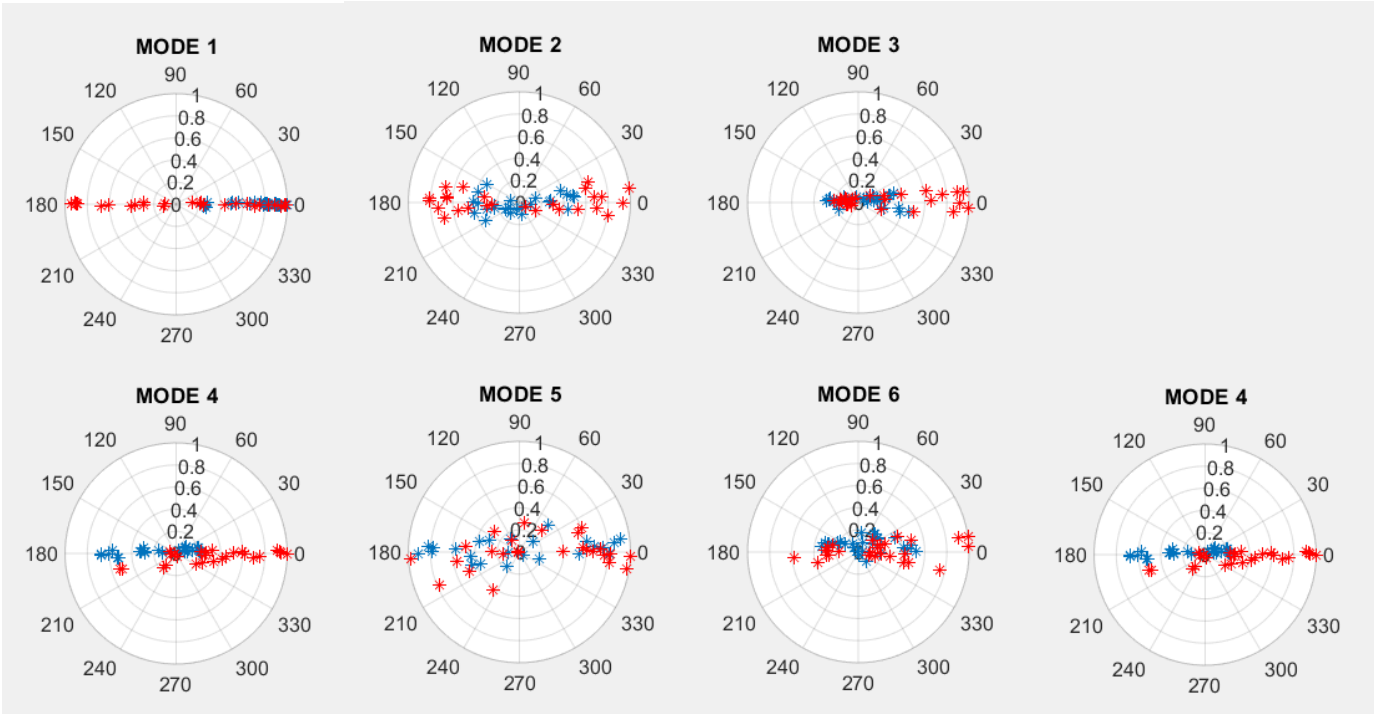

(b)

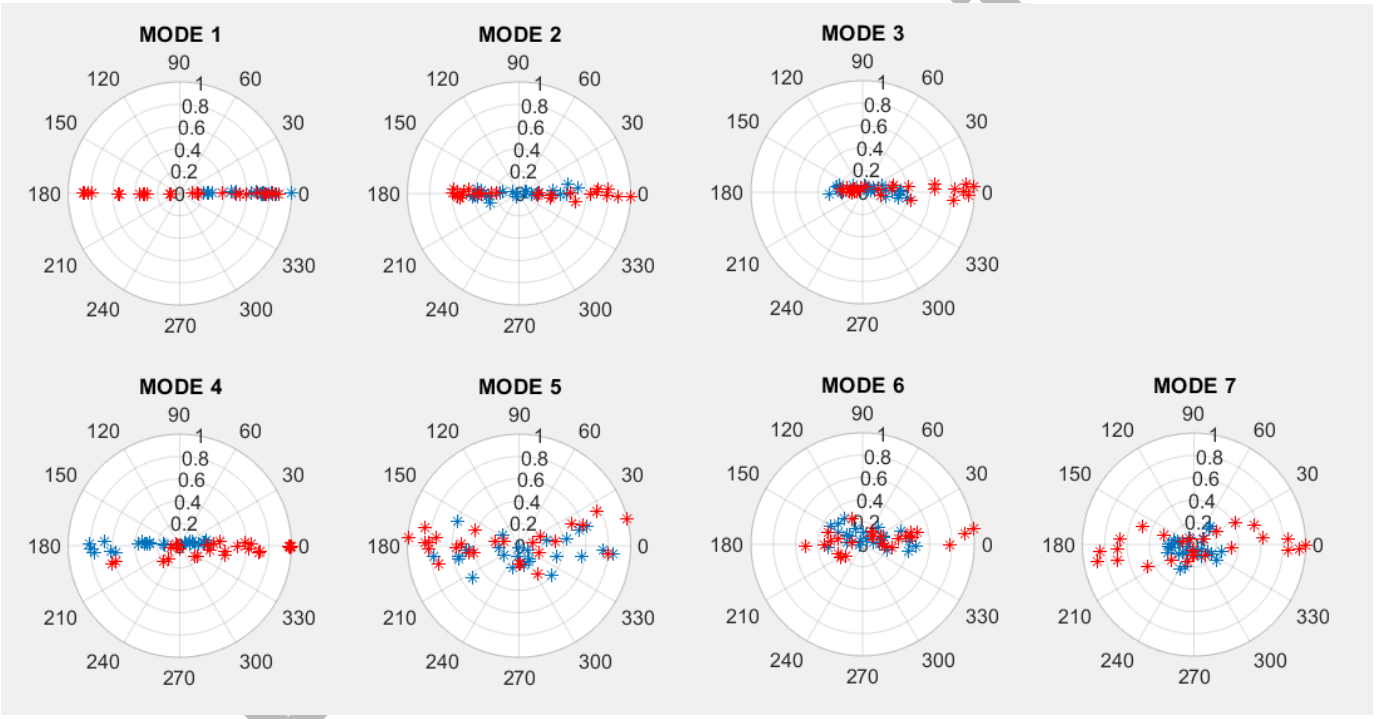

Figure 19. Mode shape components of the arch after normalization \& rotation for (a) Damage Scenario DS3 and (b) Damage Scenario DS5; $x$ components in blue and $z$ components in red. (For the interpretation of colours in the figure legend, the reader is referred to the web version of this article).

The aspects referred above are further explored by resorting to the complexity indices for damage detection proposed in the literature and applied in the numerical examples described in Section 3, namely: Modal Phase Collinearity (MPC), Modal Imaginary Ratio (MIR), Modal Dispersity (MD), Modal Phase Differences (MPD) and Modal Polygonal Area (MPA). The results obtained from these measurable indices across the different damage scenarios of the arch are displayed in Figure 20 along with the conventional Modal Complexity Factor (MCF). Since the damage caused to the masonry arch mainly affected its longitudinal and bending 
behaviour, only the in-plane vibration modes are taken into consideration, namely $\phi_{1}, \phi_{3}$ and $\phi_{4}$

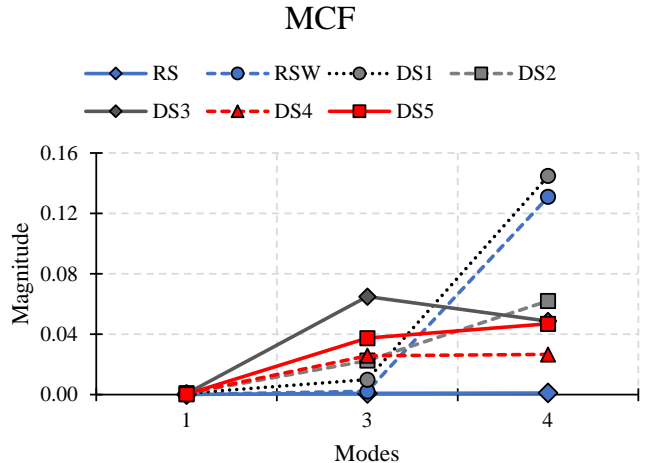

(a)

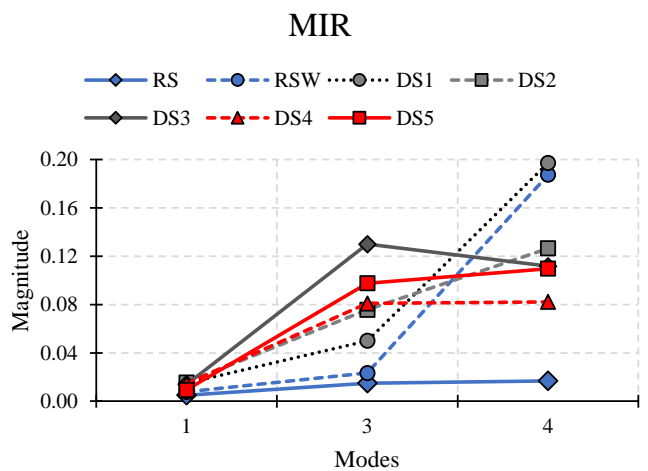

(c)

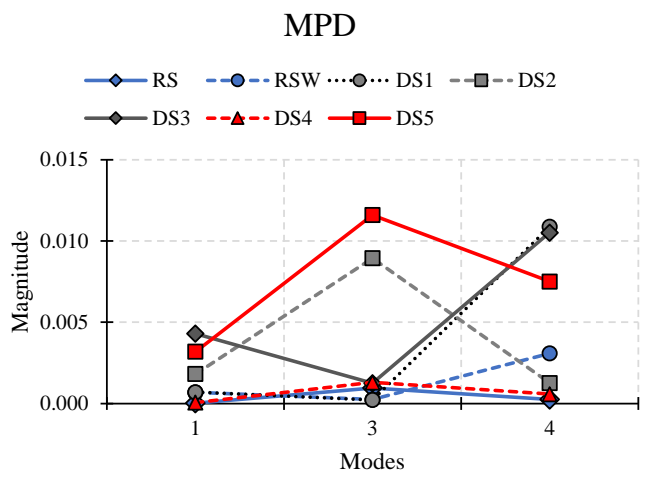

(e)

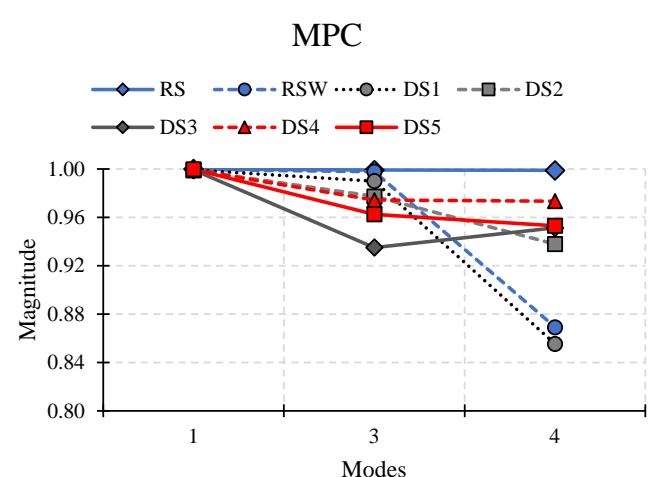

(b)

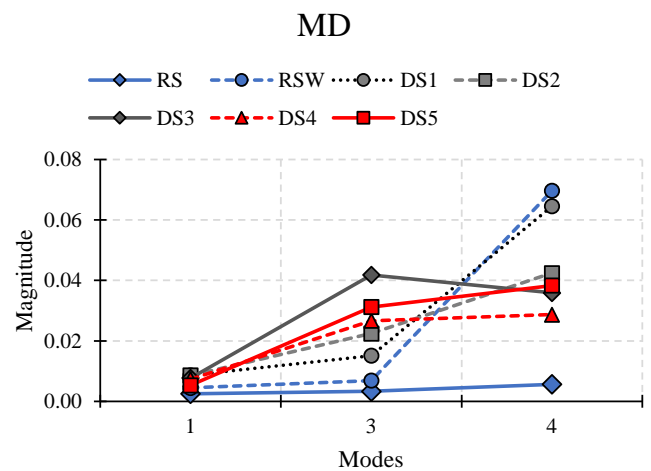

(d)

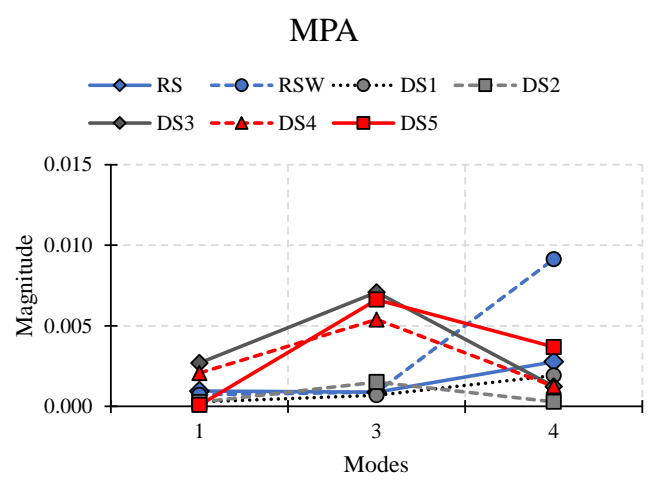

(f)

Figure 20. Evolution of MCF, MPC, MIR, MD, MPD and MPA for the in-plane mode shapes of the arch with progressive DSs. (For the interpretation of colours in the figure legend, the reader is referred to the web version of this article).

Although differently scaled in magnitude, the first four indices do share the same trend (Figure 20, $a-b-c-d)$ : in fact, the imaginary content of the modes (MIR) is proportionally linked to their complexity (MCF) and dispersity (MD), and inversely proportional to the modal phase 
collinearity (MPC). The last two indices show instead a very different sensitivity (Figure 20, $e-f)$. However, none of the indicators show consistency between their magnitude and the increasing degree of damage across the different scenarios nor provide for a unique solution. For instance, with regard to the initial undamaged configuration (RS), only two out of six indicators provide the correct outcome $(\mathrm{MCF}=0$ and $\mathrm{MPC}=1)$ while the others slightly deviate from the expected theoretical value (according to their formulation, MIR, MD, MPD and MPA should provide a zero value for undamaged and proportionally damped systems). In what concerns the reference scenario with loaded masses $\left(\mathrm{RS}_{\mathrm{W}}\right)$, most of the indices fail to address the right nature of the damping, thus the error between their actual and expected values sensibly differs. Focusing on the remaining scenarios (DSs), since the position of crack hinges remains unchanged throughout the development of the kinematic configuration [40] and considering the non-recoverability of the damage (no closure of cracks), a coherent increase of the indicators among successive DSs would be expected within each single mode. In this case, the results from MDP and MPA appear as the most inconclusive, while the others are deemed acceptable to a certain extent only for damage detection but fail in quantifying the damage (for example, the complexity index for mode $\phi_{1}$ is always very low, despite the remarkable stiffness loss experimentally observed). The major limit is that none of the analysed indicators provide information about the damage position, thereby resulting unsuitable to address higher-level damage identification problems (e.g. damage localization), which today represent one of the main challenges in structural heath monitoring.

\subsection{Pointwise damage analysis through relative variation of complex-valued modal vectors}

In order to overcome the limitations of literature indices and enhance the damage investigation procedure moving from a global to a local damage identification, the complex-valued eigenvectors describing the in-plane experimental modes of the masonry arch are thoroughly analysed and a pointwise damage indicator is proposed therefrom.

It is widely accepted that the real part of the vibration modes of a structure holds most of the information about its dynamic characteristics. Still, when local damage phenomena occur, structures are prone to experience unsynchronized movements among the different nodes, thus the real part of the modal vectors, being related to the in-phase components of the structural response, can provide only limited information about the damage position with respect to its imaginary counterpart. As a result, depending on the origin and location of the damage, indices 
relying on direct changes of real mode shapes and/or modal curvatures might lead to false negatives when the deflection characteristics of the analysed structure are not much affected by the damage [16]. By contrast, the imaginary content of the modal vectors is related to the out-of-phase components of the structural response and can be profitably exploited as a measure of damage, as shown in Section 3. In theory, the direct comparison of the magnitude of the imaginary parts is enough to quantify the degree of scatter of complex modal vectors between subsequent damage scenarios and consequently locate the damage; in reality, the process might be subtle and can lead to false alarms. As an example, Figure 21 shows the pointwise comparison of the imaginary part featured by the in-plane eigenmodes of the arch in both horizontal $(x)$ and vertical $(z)$ directions when passing from reference scenario $\mathrm{RS}_{\mathrm{W}}$ to damage scenarios DS3 (intermediate damage) and DS5 (severe damage). Although the shifts observed in the complex eigenvectors allow to detect the presence of anomalous changes, it is not possible to identify the exact location of the experimental cracks.
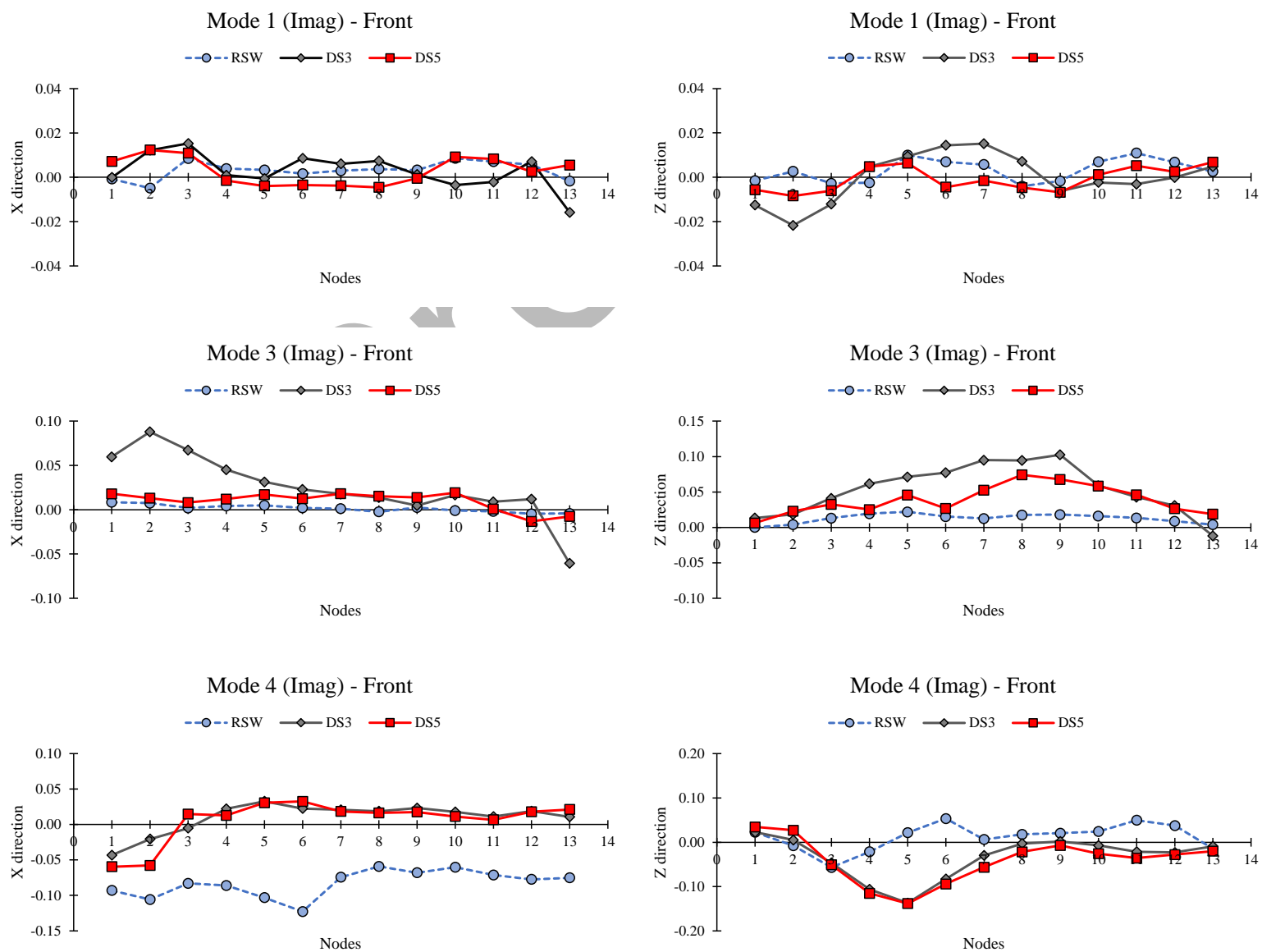

Figure 21. Pointwise comparison of the imaginary part featured by the in-plane eigenmodes of the arch along $x$ (left) and $z$ (right) directions from RSW to DS3 and DS5. (For the interpretation of colours in the figure legend, the reader is referred to the web version of this article). 
In light of the findings emerged from the numerical and experimental investigations discussed hitherto, the following damage index has been defined to appropriately compare and weigh the changes in the imaginary content of complex eigenvectors caused by evolutionary damage scenarios:

$$
\Delta \mathrm{I}_{\mathrm{ij}}\left(f_{\mathrm{j}}\right)=\left|\frac{\operatorname{Im}\left(\phi_{\mathrm{ij}}{ }^{d}\right)-\operatorname{Im}\left(\phi_{\mathrm{ij}}{ }^{u}\right)}{\mid \frac{1}{n} \sum_{\mathrm{i}=1}^{n} \operatorname{Im}\left(\phi_{\mathrm{ij}}{ }^{u}\right)}\right| \frac{f_{\mathrm{j}}{ }^{u}}{f_{\mathrm{j}}{ }^{d}}
$$

where $\operatorname{Im}(\cdot)$ is the imaginary part of the mode shape; $\phi_{\mathrm{ij}}$ is the $\mathrm{i}^{\text {th }}$ component of the $\mathrm{j}^{\text {th }}$ natural mode shape $\phi_{\mathrm{j}}$; $n$ is the total number of imaginary elements contained in the mode shape; $f_{\mathrm{j}}$ is the eigenfrequency associated with the $\mathrm{j}^{\text {th }}$ mode, and the upper scripts $u$ and $d$ stand for undamaged and damaged scenarios. Basically, $\Delta I$ is a damage indicator that measures the pointwise difference between benchmark and current magnitude of the imaginary part of a modal vector and relates this variation to its initial average imaginary content. The obtained value is then weighted by the ratio between the initial and current eigenfrequency values estimated for that mode, thus assigning higher importance to the eigenmodes suffering a significant decrease in vibration energy because of the progressive stiffness loss. If no damage occurs in the structure, the amount of changes in the imaginary content of complex-valued vectors between scenarios will be equal to zero for any $i$ and $j$, thus $\Delta \mathrm{I}_{\mathrm{j}}\left(f_{\mathrm{j}}\right)=0$. On the contrary, if damage occurs, the index in Eq. (1.22) will be different than zero for all the modes $\phi_{\mathrm{j}}(j=1$, $2, \ldots, m)$ affected by that particular damage scenario and will result into a DOF-dependent eigenvector of scalar components featuring higher peaks nearby or in correspondence of the damaged nodes, thus enabling both damage localization and quantification; each scalar is associated with a specific DOF and the dimension of the vector equals the number of experimentally measured DOFs. If data from multiple damage scenarios are available, a relative index can be computed to track the progression of damage up to the last DS. It is stressed that the interpretation of this weighted imaginary variation is univocal and insensitive to problems of modal density and data incompleteness.

The effectiveness of the proposed index is verified by comparing the results obtained from its application to the complex eigenmodes of the arch against the crack pattern experimentally observed. Given the different impact that the damage had on the in-plane deflection characteristics of the structure as well as on its longitudinal and bending stiffness (see Section 4.2), horizontal ( $x$ ) and vertical ( $z$ ) direction have been considered separately, according to their 
relevance in terms of damage effects. The results of the pointwise damage analysis are shown in Figure 22 for the modes that suffered the most significant frequency drop (i.e. $\phi_{1}$ and $\phi_{4}$ ). As it can be observed, the imaginary content variation between corresponding eigenvectors over progressive scenarios (DS3 vs RS $w$ and DS5 vs RS ) proves to be the highest in the nodes close to the position of the actual cracks. Particularly, the index allows to correctly localize the damage since the intermediate scenario (DS3 vs RS ${ }_{W}$ ), featuring major peaks in the vicinity of cracks $c_{2}$ and $c_{3}$ for mode $\phi_{1}$, i.e. the mode influenced by the formation of crack hinges at the supports, and in correspondence of crack $c_{1}$ for mode $\phi_{4}$, i.e. the mode mainly affected by the crack hinge occurred near the keystone.

Finally, the index is computed by considering the relative imaginary variation of all in-plane complex vectors, each one weighted by the respective frequency ratio, in both horizontal and vertical direction. The outcome of this step is reported in Figure 23. It is shown that, in absolute terms, the comparison of both DSs with the $\mathrm{RS}_{\mathrm{w}}$ allows to identify as damaged DOFs only the nodes close to crack $c_{1}$, whereas the relative comparison between progressive DSs enables to catch the three damage locations as well as the experimental crack sequence.

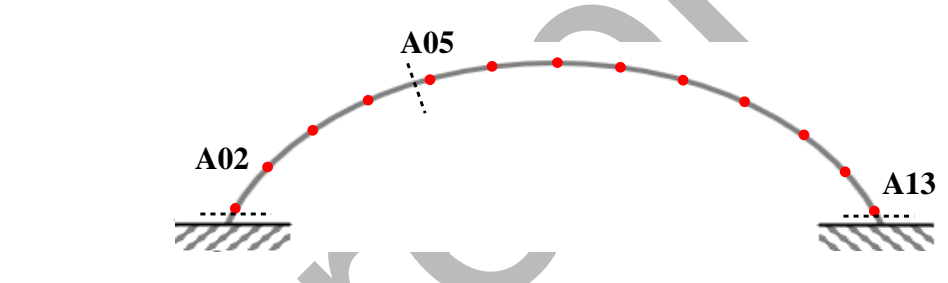

Mode 1 - DS3 vs RSW

$\square \mathrm{X}$ front $\square \mathrm{X}$ back

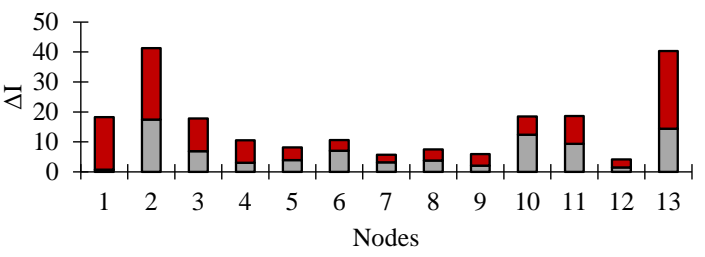

Mode 1 - DS5 vs RSW

$\square \mathrm{X}$ front $\square \mathrm{X}$ back

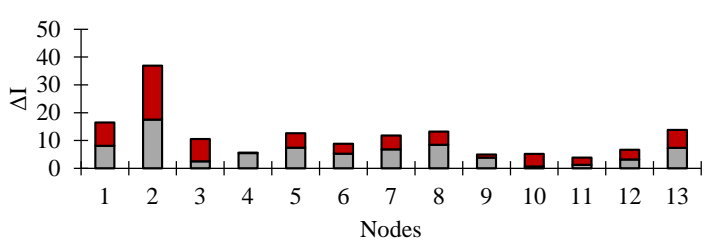

Mode 4 - DS3 vs RSW

$\square \mathrm{Z}$ front $\square \mathrm{Z}$ back

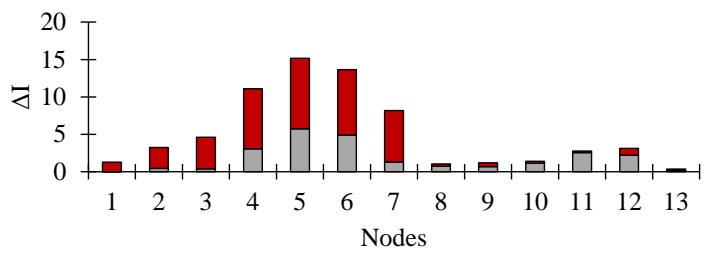

Mode 4 - DS5 vs RSW $\square \mathrm{Z}$ front $\square \mathrm{Z}$ back

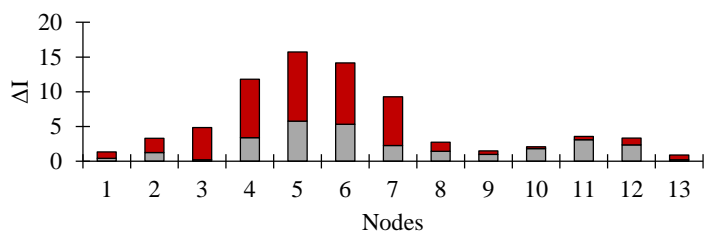

Figure 22.Results of the pointwise damage analysis through the proposed index $\Delta \mathrm{I}$ based on single mode contribution: localization and quantification of damage over progressive scenarios along the front and back edges of the arch in $x$ (left) and $z$ (right) direction. (For the interpretation of colours in the figure legend, the reader is referred to the web version of this article). 

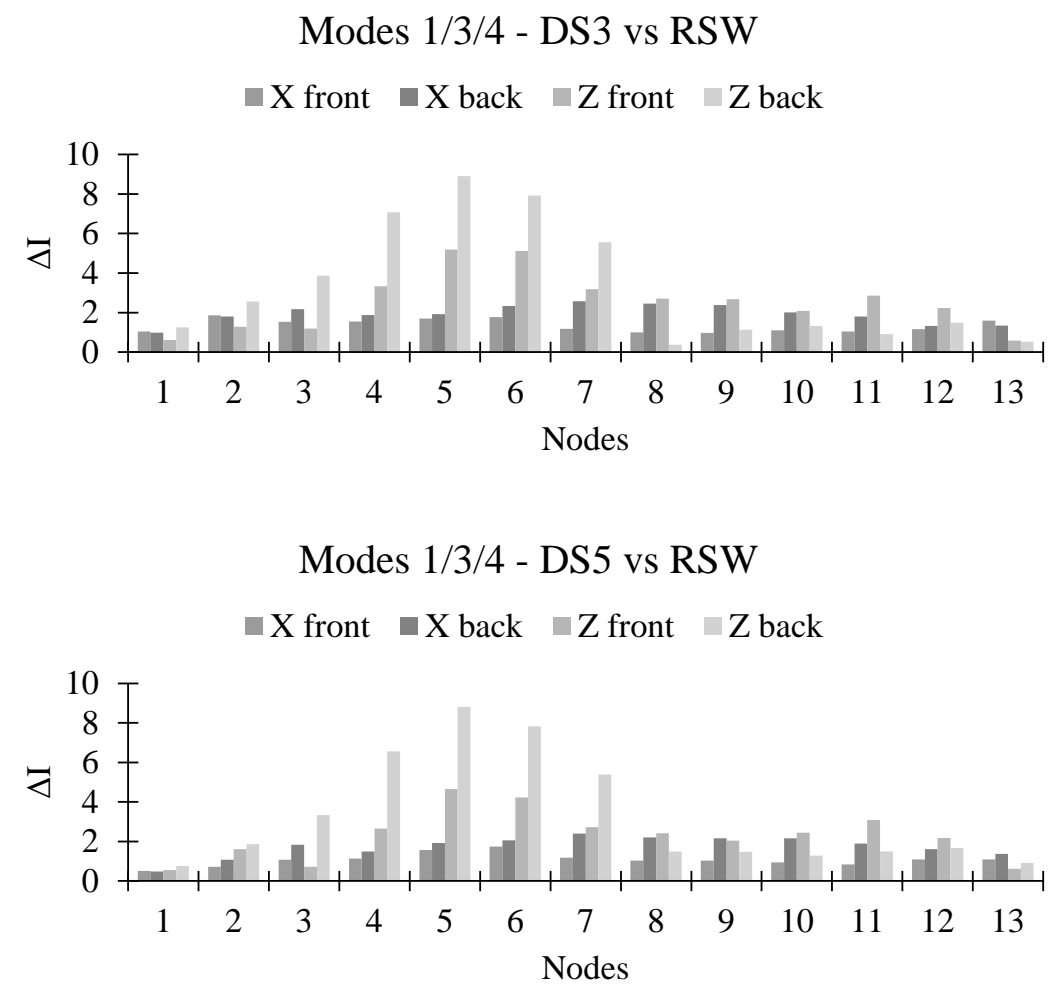

Modes 1/3/4 - DS5 vs DS3

$\square$ X front $\square$ X back $\square$ Z front $\square$ Z back

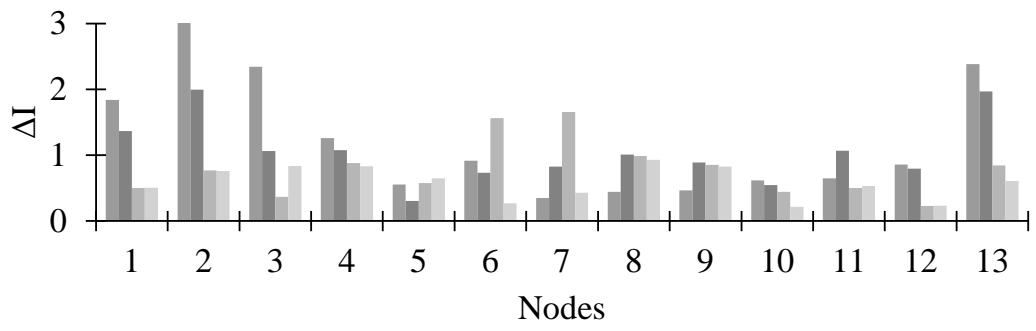

Figure 23. Results of the pointwise damage analysis through the proposed index $\Delta \mathrm{I}$ based on multimode contribution: localization and quantification of damage over progressive scenarios along the front and back edges of the arch in $x$ and $z$ directions. (For the interpretation of colours in the figure legend, the reader is referred to the web version of this article).

\section{Conclusions}

The present paper investigated the role and evolution of complex mode shapes in damaged systems featuring nonproportional damping from a numerical and experimental point of view. For this purpose, two numerical examples were firstly analysed: a pinned beam and a segmental arch on fixed supports both subjected to increasing damage scenarios. In either case, except for the modal phase difference - MPD, all state-of-the-art modal complexity indices employed for 
damage detection proved to be very sensitive to damage (in particular for higher-order mode shapes) and to increase monotonically with the damage evolution, but they resulted unable to furnish information about the damage location. In fact, the close analysis of both the real and imaginary part of the complex mode shapes over the different scenarios showed that damageinduced shifts were almost undetectable in the real part, while the imaginary component resulted very sensitive to structural damage, increasing with the damage severity and reaching its maximum value near the affected areas. Afterwards, the relationship between damage and complex-valued modal vectors was further investigated making use of the experimental data from a real physical system, i.e. a reduced-scale arch specimen subjected to progressive settlement-induced damage scenarios. The analyses allowed to uncover the numerous challenges associated with the modal identification of real structures as well as the difficulties that arise in identifying the damage when available state-of-the-art formulations based on modal complexity are applied. Hence, to overcome the limitations that literature indices exhibit for experimental use, a pointwise index relying on the imaginary content variation of measured complex modes is proposed and applied to the tested arch for both localization and assessment of structural damage. In many years of publications about modal complexity, it has been shown that no index could be considered universally effective for damage detection using complex modes. However, this study demonstrated that tracking the variation of complex mode shapes not only enables to detect the presence of damage, but also to localize, quantify, and follow its evolution, which is of crucial importance for the monitoring and assessment of strategic structures and infrastructure systems. The results obtained via the proposed damage index proved to be in good agreement with the experimental evidence, showing its potential suitability as a damage identification tool for complex and nonhomogeneous real-world structures featuring nonproportional damping. Still, given the difficulty of the subject, further research must be carried out on the full-scale systems to generalize the conclusions.

\section{CRediT authorship contribution statement}

All authors listed have made a substantial, direct, and intellectual contribution to the work, and approved it for publication. 


\section{Acknowledgements}

The present study has been carried out within the framework of the National Operational Programme on Research and Innovation (Attraction and International Mobility) PON-AIM 2014-2020 Line 2, co-financed by the European Social Fund and by the National Rotation Fund.

\section{References}

[1] M. Vasta, Classically and Nonclassically Damped Multi-degree of Freedom (MDOF) Structural Systems, Dynamic Response Characterization of, in: M. Beer, I. Kougioumtzoglou, E. Patelli, IK. Au (Eds.), Encyclopedia of Earthquake Engineering. Springer, Berlin, Heidelberg, 2014. https://doi.org/10.1007/978-3-642-36197-5_159-1

[2] S.R. Ibrahim, Existence and Normalization of Complex Modes for Post Experimental Use in Modal Analysisc, in: Silva J.M.M., Maia N.M.M. (Eds.) Modal Analysis and Testing. NATO Science Series (Series E: Applied Sciences), vol 363, Springer, Dordrecht, 1999. https://doi.org/10.1007/978-94-011-4503-9_21

[3] K. Liu, M.R. Kujath, W. Zheng, Quantification of non-proportionality of damping in discrete vibratory systems, Computers and Structures. 77 (2000) 557-569. https://doi.org/10.1016/S0045-7949(99)00230-8

[4] U. Fuellekrug, Computation of real normal modes from complex eigenvectors, Mechanical Systems and Signal Processing. 22 (2008) 57-65. https://doi.org/10.1016/j.ymssp.2007.07.009

[5] M.L. Wei, R.J. Allemang, D.L. Brown, Real normalization of measured complex modes, in: Proc. of the $5^{\text {th }}$ International Modal Analysis Conference, London, UK, 1987, pp. 708-712.

[6] F. Lembregts, M. Brughmans, Estimation of real modes from FRFs via direct parameter identification, in: Proc. of the $7^{\text {th }}$ International Modal Analysis Conference, Las Vegas, NV, USA, 1989, pp. 631-636.

[7] F. Lembregts, J. Leuridan, H. van Brussel, Frequency domain identification techniques for modal analysis: state space formulation, Mechanical Systems and Signal Processing. 4:1 (1990) 65-75. https://doi.org/10.1016/0888-3270(90)90041-I 
[8] M.I. Friswell, J.E. Mottershead, Finite Element Model Updating in Structural Dynamics, Kluwer Academic Publishers, Dordrecht, Boston, London, 1995. https://doi.org/10.1007/978-94-015-8508-8

[9] E. Balmes, New results on the identification of normal modes from experimental complex modes, Mechanical Systems and Signal Processing. 11:2 (1997) 229-243. https://doi.org/10.1006/mssp.1996.0058

[10] D.J. Ewins, Modal Testing: Theory, Practice and Application, second ed., Research Studies Press Ltd., Baldock, Hertfordshire, England, 2000.

[11] G. Gloth, M. Degener, U. Fuellekrug, J. Gschwilm, M. Sinapius, P. Fargette, B. Levadoux, P. Lubrina, New ground vibration testing techniques for large aircraft, Sound and Vibration. 35 (11) (2001) 14-18.

[12] K.M. Ahmida, J.R.F. Arruda, On the relation between complex modes and wave propagation phenomena, Journal of Sound and Vibration. 255:4 (2002) 663-684. https://doi.org/10.1006/jsvi.2001.4183

[13] H. Koruk and K.Y. Sanliturk, A novel definition for quantification of mode shape complexity, Journal of Sound and Vibration. 332:14 (2013) 3390-3403. https://doi.org/10.1016/j.jsv.2013.01.039

[14] M.G. Masciotta, L.F. Ramos, P.B. Lourenço, M. Vasta, Spectral algorithm for nondestructive damage localisation: Application to an ancient masonry arch model, Mechanical Systems and Signal Processing 84 (2017) 286-307. https://doi.org/10.1016/j.ymssp.2016.06.034

[15] B. Gunes, O. Gunes: Structural health monitoring and damage assessment part I: A critical review of approaches and methods, Int. J. Phys. Sci. 8:34 (2013) 1694-1702. https://doi.org/10.5897/IJPS11.1327

[16] M.G. Masciotta, L.F. Ramos, P.B. Lourenço, M. Vasta, G. De Roeck, A spectrumdriven damage identification technique: Application and validation through the numerical simulation of the Z24 Bridge, Mechanical Systems and Signal Processing. 70-71 (2016) 578-600. https://doi.org/10.1016/j.ymssp.2015.08.027

[17] S. Vanlanduit, E. Parloo, B. Cauberghe, P. Guillaume, P. Verboven, A robust singular value decomposition for damage detection under changing operating conditions and structural uncertainties, J. Sound Vib. 284:3-5 (2005) 1033-1050.

https://doi.org/10.1016/j.jsv.2004.07.016

[18] G. Kawiecki, Modal damping measurement for damage detection, Smart Mater Struct. 10(3) (2001) 466-471. 
[19] E.I. Egba, Detection of structural damage in building using changes in modal damping mechanism, Int. J. Eng. Manag. Sci. 3(3) (2012) 250-255.

[20] R.O. Curadelli, J.D. Riera, D. Ambrosini, M.G. Amani, Damage detection by means of structural damping identification, Eng. Struct. 30(2008) 3497-3504. https://doi.org/10.1016/j.engstruct.2008.05.024

[21] E. Lofrano, A. Paolone, G. Ruta, 2020. Dynamic damage identification using complex mode shapes, Structural Control and Health Monitoring, 27(12), e2632. https://doi.org/10.1002/stc.2632

[22] C. Valente, D. Spina, S. Gabriele, A. De Leonardis, The Complex Plane Representation Method for Structural Damage Detection, in: B.H.V. Topping, J.M. Adam, F.J. Pallarés, R. Bru, M.L. Romero (Eds.), Proc. of the $10^{\text {th }}$ International Conference on Computational Structures Technology, Civil-Comp Press, Stirlingshire, UK, Paper 56, 2010. https://doi:10.4203/ccp.93.56

[23] F. Iezzi, D. Spina, C. Valente, 2015. Damage assessment through changes in mode shapes due to non-proportional damping, J Phys: Conf Ser. 628, 012019. https://doi:10.1088/1742-6596/628/1/012019

[24] F. Iezzi, C. Valente, Modal density influence on modal complexity quantification in dynamic systems, Procedia Eng. 199(2017) 942-947.

\section{https://doi.org/10.1016/j.proeng.2017.09.245}

[25] F. Deblauwe, R.J. Allemang, A possible origin of complex modal vectors, in: Proc. of the $11^{\text {th }}$ International Seminar on Modal Analysis, paper A2-3, Katholic University of Leuven, 1986.

[26] T. K. Caughey, M. E. J. O’Kelly, Classical normal modes in damped linear dynamic systems, J. Appl. Mech. Trans. ASME 32(1965) 583-588.

[27] A.M. Kabe, B.H. Sako, Structural Dynamics Fundamentals and Advanced Applications, Volume I, ISBN: 978-0-12-821614-9.

[28] K.J. Bathe and E.L. Wilson, Numerical methods in finite element analysis. Upper Saddle River: Prentice-Hall, 1976.

[29] D. S. Mackey, N. Mackey, C. Mehl, V. Mehrmann, Vector spaces of linearizations for matrix polynomials. SIAM Journal on Matrix Analysis and Applications, 28:4 (2006) 971-1004. https://doi.org/10.1137/050628350

[30] G. Prater Jr, and R. Singh, Quantification of the extent of non-proportional viscous damping in discrete vibratory systems, Journal of Sound and Vibration. 104:1 (1986) 109-125. https://doi.org/10.1016/S0022-460X(86)80134-1 
[31] R.S. Pappa, K.B. Elliott, A. Schenk, Consistent-mode indicator for the eigensystem realization algorithm, Journal of Guidance, Control, and Dynamics, $16: 5$ (1993) 852858. https://doi.org/10.2514/3.21092

[32] R.J. Allemang, D.L. Brown, A correlation coefficient for modal vector analysis, in: Proc. of the $1^{\text {st }}$ International Modal Analysis Conference, Orlando, FL, 1982, pp.110116.

[33] P. Vacher, B. Jacquier, A. Bucharles, Extensions of the MAC criterion to complex modes, in: Proc. of ISMA2010 including USD2010, Leuven, 2010, pp. 2713-2725.

[34] J.W.S. Rayleigh, 1945. Theory of Sound, vol. I. Dover Publications, New York, New York, 1877.

[35] M. Girardi, C. Padovani, D. Pellegrini, The NOSA-ITACA code for the safety assessment of ancient constructions: A case study in Livorno, Adv. Eng. Softw. 89 (2015) 64-76. https://doi.org/10.1016/j.advengsoft.2015.04.002

[36] M. Girardi, C. Padovani, D. Pellegrini, M. Porcelli, L. Robol, 2020. Finite element model updating for structural applications, J. Comput. Appl. Math. 370, 112675. https://doi.org/10.1016/j.cam.2019.112675

[37] M. Girardi, C. Padovani, D. Pellegrini, L. Robol, 2019. Model updating procedure to enhance structural analysis in FE code NOSA-ITACA, J. Perform. Construct. Fac. 33:4, 04019041. https://doi.org/10.1061/(ASCE)CF.1943-5509.0001303

[38] M. Girardi, C. Padovani, D. Pellegrini et al., 2021. A finite element model updating method based on global optimization, Mechanical Systems and Signal Processing, 152, 107372. https://doi.org/10.1016/j.ymssp.2020.107372

[39] V. Binante, M. Girardi, C. Padovani, G. Pasquinelli, D. Pellegrini, M. Porcelli, L. Robol, Nosa-itaca 1.1 documentation, 2017.

[40] M.G. Masciotta, D. Pellegrini, D. Brigante, A. Barontini, P.B. Lourenço, M. Girardi, C. Padovani, G. Fabbrocino, Dynamic characterization of progressively damaged segmental masonry arches with one settled support: experimental and numerical analyses, Frattura ed Integrità Strutturale, 14:51 (2020) 423-441.

https://doi.org/10.3221/IGF-ESIS.51.31 